\title{
The First Difference Property of the Present Value Operator
}

\author{
Buser, Stephen A.; Jensen, Bjarne Astrup
}

Document Version

Accepted author manuscript

Published in:

Quarterly Journal of Finance

DOI:

$10.1142 / \mathrm{S} 2010139217500124$

Publication date:

2017

License

Unspecified

Citation for published version (APA):

Buser, S. A., \& Jensen, B. A. (2017). The First Difference Property of the Present Value Operator. Quarterly Journal of Finance, $7(4)$, [1750012]. https://doi.org/10.1142/S2010139217500124

Link to publication in CBS Research Portal

\section{General rights}

Copyright and moral rights for the publications made accessible in the public portal are retained by the authors and/or other copyright owners and it is a condition of accessing publications that users recognise and abide by the legal requirements associated with these rights.

Take down policy

If you believe that this document breaches copyright please contact us (research.lib@cbs.dk) providing details, and we will remove access to the work immediately and investigate your claim. 


\section{The First Difference Property of the Present Value Operator}

\section{Stephen A. Buser and Bjarne Astrup Jensen}

Journal article (Accepted manuscript*)

\section{Please cite this article as:}

Buser, S. A., \& Jensen, B. A. (2017). The First Difference Property of the Present Value Operator. Quarterly Journal of Finance, 7(4), [1750012]. D0l: 10.1142/\$2010139217500124

DOI: 10.1142/\$2010139217500124

* This version of the article has been accepted for publication and undergone full peer review but has not been through the copyediting, typesetting, pagination and proofreading process, which may lead to differences between this version and the publisher's final version AKA Version of Record.

Uploaded to CBS Research Portal: January २019 


\begin{abstract}
This paper identifies fundamental relationships between the present value of a given cash flow and the present value of the first as well as second order changes in that cash flow. These relations apply only simple arithmetic operations and avoids the use of differential calculus and advanced series summation in order to derive analytic expressions for present values, duration and convexity of a variety of cash flows; expressions that relate the quantitative outcomes of numerical calculations to the driving forces behind those calculations.

We apply the methods to a variety of nontraditional cash flows, including cash flows with polynomial growth, cash flows that are subject to different tax effects for dividends and capital gain and cash flows that exhibit cyclical variation over time.
\end{abstract}

Keywords: Present value, first and second order difference property, recursive calculation, duration, convexity 


\section{Introduction}

The present value of a future cash flow, together with derived risk measures such as duration and convexity, are some of the most fundamental concepts in all of financial economics. Although technological advances have greatly enhanced methods for numerical calculations of these values, analytic solutions continue to play an important role by identifying essential properties of such calculations and the driving forces behind them.

One standard textbook approach initially calculates the discounted value of future payments and then scales those values by the maturity date for the payment, in the case of duration, and by the square of the maturity date, in the case of convexity. An alternative approach identifies the first and second derivative for a given analytic formula for the present value and then uses those expressions to quantify measures for duration and convexity. That approach is the one that was almost universally used in earlier papers from the 1970'es and 1980'es $1^{1}$ but it is limited to cases in which pre-existing expressions have been identified for present value.

Notably, however, closed form expressions are rarely, if ever, found in standard textbooks on corporate finance, fixed income and investment analysis as of today, where it is common to present these concepts with one or a few simple numerical examples without proper illustration of the sensitivity of these magnitudes to, e.g., the level of the interest rate or the maturity of the cash flow in question. One reason may be that these traditional approaches can become tedious and typically feature mathematical challenges in themselves.

In this paper we show that for a variety of cash flows there is a simple mathematical approach to reach analytic expressions for the present value and the derived risk measures. We show that two surprisingly simple properties of the general present value equation can be used to derive analytic expressions for present value, duration and convexity for a broad class of payment streams.

This approach does not involve advanced mathematical knowledge of series summation or the ability to differentiate complicated formulas. It only involves the application of simple arithmetic operations applied to differences of the cash flow rather than to the cash flows themselves. In this manner, the paper also has a pedagogical angle.

The remainder of the paper is organized as follows. In Section 2 we identify the specific properties of the present value operator that leads to each and every result presented in the paper. In Section 3 we show how applications of the general property confirm the

\footnotetext{
${ }^{1}$ For a brief historical overview of earlier contributions we refer to Section 2 in Buser and Jensen (2017).
} 
traditional present value rules for familiar payment streams. Section 4 demonstrates that the general property can be applied to identify analytic expressions for payment streams that are less familiar but potentially useful. Examples include payment streams that increase at polynomial rates over time and payment streams with cyclical variation. We also show an example that describes the relationship between yields before tax and yields after tax for an investor in a tax regime based on the realization principle. Sections 5 and 6 demonstrate that the same general approach can be used to identify analytic expressions for duration and convexity for a broad class of cash flows. Proofs of the respective findings are provided in the Appendices.

\section{The First and Second Difference Properties}

Consider payment streams that are denoted as:

$$
\underline{C} \equiv\left\{C_{1}, C_{2}, \ldots, C_{t}, \ldots, C_{T}, \ldots\right\}
$$

This definition covers perpetual as well as finite payment streams with $C_{T}$ being the last payment, in which case $C_{T+1}=C_{T+2}=\ldots 0$. In the following we will consistently write $T$ as the last payment date and read this as $T=\infty$ in case of a perpetual payment stream.

Definition 1. For a given payment stream $\underline{C}$ and a given rate of interest $r$, the present value of the payment stream is defined as:

$$
V(\underline{C} ; r, T) \equiv \sum_{t=1}^{T} C_{t}(1+r)^{-t}
$$

Definition 2. For a given payment stream, $\underline{C}$, the first difference in payments from time $t$ to time $t+1$ is denoted by $\Delta_{t}$ and defined as:

$$
\Delta_{t} \equiv C_{t+1}-C_{t}, t=1,2, \ldots T
$$

with the convention that $\Delta_{T} \equiv-C_{T}$ when $T<\infty$. 
Proposition 1. For any payment stream, $\underline{C}$, the relationship between the present value of the payment stream and the present value of its first difference can be expressed as:

$$
r V(\underline{C} ; r, T)=C_{1}+\sum_{t=1}^{T} \Delta_{t}(1+r)^{-t}
$$

Proof. A proof of Proposition 1 is provided in Appendix A.1.

We subsequently refer to the relationship in (4) as the First Difference Property, which we hereafter abbreviate as FDP.

\section{Remark 1.}

One interpretation of Equation (4) is in terms of valuation by the constant yield to maturity principle or in terms of "true economic depreciation" $\square^{2}$ The yield $r$ earned over the first period on the investment outlay $V(\underline{C} ; r, T)$, i.e. $r V(\underline{C} ; r, T)$, is composed of two terms: Receipt of the first payment $C_{1}$ together with a simultaneous adjustment for the change in the present value:

$$
C_{1}+\underbrace{\sum_{t=2}^{T} C_{t}(1+r)^{-(t-1)}}_{\text {time } 1 \text { value of remaining payments }}-\underbrace{\sum_{t=1}^{T} C_{t}(1+r)^{-t}}_{\text {time } 0 \text { value of payments }}=C_{1}+\sum_{t=1}^{T} \Delta_{t}(1+r)^{-t}
$$

I Similar to the FDP we can define second differences as:

$$
\left.\Delta_{t}^{2} \equiv \Delta_{t+1}-\Delta_{t}=C_{t+2}-C_{t+1}-\left(C_{t+1}-C_{t}\right)\right)=C_{t+2}-2 C_{t+1}+C_{t}
$$

Proposition 2. For any payment stream, $\underline{C}$, the relationship between the present value of the payment stream and the present value of its first difference can be expressed as:

$$
r V(\underline{C} ; r, T)=C_{1}+\frac{1}{r}\left[\Delta_{1}+\sum_{t=1}^{T} \Delta_{t}^{2}(1+r)^{-t}\right]
$$

\footnotetext{
${ }^{2}$ Cf. Samuelson's classical paper on true economic depreciation, Samuelson 1964).
} 
We subsequently refer to the relationship in (7) as the Second Difference Property, which we hereafter abbreviate as SDP.

\section{Applications of the FDP and SDP to Familiar Cash Flow Patterns}

In this section we demonstrate some examples of how the difference properties produce well known results in a simple manner.

Example 1: Annuity payments

For the annuity with payments at a constant level $C$ the first differences in the cash flow vanish, except for the final term:

$$
\Delta_{t}=\left\{\begin{array}{cl}
0 & \text { for } t=1,2, \ldots, T-1 \\
-C & \text { for } t=T
\end{array}\right.
$$

Hence, the FDP expression gives the usual annuity formula:

$$
r V(\underline{C} ; r, T)=C-C(1+r)^{-T} \Rightarrow V(\underline{C} ; r, T)=C \frac{1-(1+r)^{-T}}{r},
$$

with limiting value $C / r$ for the perpetuity, where $T=\infty$. The latter is also the immediate result from Equation (4), since $\Delta_{t}=0 \forall t$ when $T=\infty$.

Usually, the value of a perpetual payment stream is easier to handle than its finite horizon counterpart because of the special treatment of $\Delta_{T}$. The annuity is one example, where it is not necessary to consider the finite horizon separately. The annuity with maturity $T$ can be regarded as the difference between a perpetuity and a perpetuity forward starting with the first payment at time $T+1$. For the latter, the value must be discounted back to time zero with the discount factor $(1+r)^{-T}$ :

$$
V(\underline{C} ; r, T)=\frac{C}{r}-\frac{C}{r}(1+r)^{-T}=C a_{\overline{T r}} .
$$

Here and in the following we will use the common actuarial notation for the value of an annuity:

$$
a_{\bar{T} r} \equiv \frac{1-(1+r)^{-T}}{r}
$$


Using the SDP instead, we have the following relation:

$$
\Delta_{t}^{2}=\left\{\begin{aligned}
0 & \text { for } t=1,2, \ldots, T-2 \\
-C & \text { for } t=T-1 \\
C & \text { for } t=T
\end{aligned}\right.
$$

with a result identical to Equation (9):

$$
\begin{aligned}
r V(\underline{C} ; r, T) & =C+\frac{1}{r}\left[C(1+r)^{-T}-C(1+r)^{-(T-1)}\right] \Rightarrow \\
V(\underline{C} ; r, T) & =C\left[\frac{1}{r}+\frac{(1+r)^{-T}}{r^{2}}(1-(1+r))\right]=C \frac{1-(1+r)^{-T}}{r} .
\end{aligned}
$$

Example 2: Payment streams with a constant percentage growth

Payment streams that exhibit a constant percentage growth are frequently encountered and a familiar concept in corporate finance and valuation. Other applications of the model include applications to bonds with variable coupons that are indexed to measures of inflation, but which typically assume a constant rate of appreciation for the purpose of initial valuation $3^{3}$ The model can also be applied to currency conversions, where exchange rates are assumed to change at a constant percentage rate each period. For any such case, the schedule for future cash flows is given as:

$$
C_{t}=C(1+g)^{t-1}
$$

If the assumed schedule for cash flows is projected in perpetuity, both first and second differences are proportional to the payment stream itself:

$$
\begin{aligned}
& \Delta_{t}=C(1+g)^{t}-C(1+g)^{t-1}=C(1+g)^{t-1}((1+g)-1)=g C_{t} \\
& \Delta_{t}^{2}=\Delta_{t+1}-\Delta_{t}=g \Delta_{t}=g^{2} C_{t}
\end{aligned}
$$

\footnotetext{
${ }^{3}$ For a survey of the principles used in the design of inflation-linked bonds see, e.g., Deacon and Derry (2004).
} 
Hence, using first differences we have the well-known formula: $\mathrm{H}^{4}$

$$
r V(\underline{C} ; r, \infty)=C+g V(\underline{C} ; r, \infty) \Rightarrow V(\underline{C} ; r, \infty)=\frac{C}{r-g},
$$

and similarly by using second differences:

$$
\begin{gathered}
r V(\underline{C} ; r, \infty)=C+C \frac{g}{r}+\frac{g^{2}}{r} V(\underline{C} ; r, \infty) \Rightarrow \\
\left(\frac{r^{2}-g^{2}}{r}\right) V(\underline{C} ; r, \infty)=C\left[1+\frac{g}{r}\right]=C \frac{r+g}{r} \Rightarrow V(\underline{C} ; r, \infty)=\frac{C}{r-g} .
\end{gathered}
$$

If the growth is considered for a finite time horizon only, we can consider the value as the difference between two infinite payment streams, where one of them is forward starting at time $T+1$ at the initial level $C(1+g)^{T}$. Hence, the present value is:

$$
V(\underline{C} ; r, T)=\frac{C}{r-g}-\frac{C(1+g)^{T}}{r-g}(1+r)^{-T}=C \frac{1-\left(\frac{1+r}{1+g}\right)^{-T}}{r-g} .
$$

Example 3: Bullet bonds with fixed coupon rate

A bullet bond with fixed coupon rate $c$ has the payment stream

$$
C_{t}=c, t=1,2, \ldots, T-1 ; \quad C_{T}=1+c .
$$

In this case the sequence of first differences produces two non-zero terms:

$$
\Delta_{t}= \begin{cases}0 & \text { for } t=1,2, \ldots, T-2 \\ 1 & \text { for } t=T-1 \\ -(1+c) & \text { for } t=T\end{cases}
$$

The resulting price formula includes the 0-coupon bond, where $c=0$, in which case the discount factor $(1+r)^{-T}$ is the only resulting item:

$$
\begin{aligned}
r V(\underline{C} ; r, T) & =c+(1+r)^{-(T-1)}-(1+c)(1+r)^{-T}=\left[c+(1+r)^{-T}(r-c)\right] \Leftrightarrow \\
V(\underline{C} ; r, T) & =\left[\frac{c}{r}+\left(1-\frac{c}{r}\right)(1+r)^{-T}\right]=\left[c a_{T r}+(1+r)^{-T}\right] .
\end{aligned}
$$

\footnotetext{
${ }^{4}$ Usually referred to as Gordon's growth formula with reference to Gordon and Shapiro (1956) and Gordon (1959), although the formula is already developed in Williams (1938).

${ }^{5}$ The sequence of second differences produces three non-zero terms and is omitted for this example.
} 
The first version of the pricing formula in Equation 22 is a special case of what is known in the literature as Makeham's formula, cf., e.g., Makeham (1875), Hossack and Taylor (1975), Jensen (2013) and Magni (2014). The last part of Equation (22) demonstrates that the present value of the bond is the sum of two terms. If the bond is "stripped" into an annuity of coupon payments and a pure discount bond maturing at time $T$, the first term is the present value of the coupon payments. The latter term is the present value of the remaining discount bond.

For $T=\infty$ this payment stream becomes the perpetuity with limiting value $c / r$. Again, this is also the immediate result from (4), since $\Delta_{t}=0 \forall t$.

For the bullet bond, the simple approach of interpreting the payment stream as a difference between a payment stream with an infinite maturity and a similar forward starting payment stream is not possible.

Example 4: An alternative FDP relationship

If the payment stream is generated from a fixed rate coupon bond with coupon rate $c$ and a given profile for principal repayments, which we denote as $\left(Z_{1}, Z_{2}, \ldots, Z_{n}\right)$, we can express the FDP in terms of the repayment profile. We normalize with no loss of generality these repayments such that $\sum_{j=1}^{n} Z_{j}=1$. It then holds by definition that:

$$
\begin{aligned}
& C_{t}=c\left(\sum_{j=t}^{n} Z_{j}\right)+Z_{t}, \quad C_{t+1}=c\left(\sum_{j=t+1}^{n} Z_{j}\right)+Z_{t+1} \\
& \Delta_{t}=C_{t+1}-C_{t}=Z_{t+1}-(1+c) Z_{t} .
\end{aligned}
$$

Inserting this relationship into the general FDP present value formula (4) and rearranging terms results in the expressions in Equation (24):

$$
\begin{aligned}
V(\underline{C} ; r, n) & =\frac{1}{r}\left[C_{1}+\sum_{t=1}^{n}\left(Z_{t+1}-(1+c) Z_{t}\right)(1+r)^{-t}\right] \\
& =\frac{1}{r}\left[c+Z_{1}+\sum_{t=1}^{n}\left(Z_{t+1}-(1+c) Z_{t}\right)(1+r)^{-t}\right] \\
& =\frac{1}{r}\left[c\left(1-\sum_{t=1}^{n} Z_{t}(1+r)^{-t}\right)+\sum_{t=0}^{n}\left(Z_{t+1}-Z_{t}\right)(1+r)^{-t}\right] .
\end{aligned}
$$

We will make use of this way of writing the FDP formula later on in Section 4.3. Here we show three simple examples of how this variant of the FDP relationship works: 
- For the bullet bond with unit face value, Equation (24) becomes

$$
V(\underline{C} ; r, n)=\frac{1}{r}\left[c+(1+r)^{-(n-1)}-(1+c)(1+r)^{-n}\right]=c a_{\bar{n} r}+(1+r)^{-n},
$$

in accordance with Equation (22).

- For the annuity we have that the repayments follow a geometric series: $Z_{t+1}=(1+c) Z_{t}$. Hence, all terms in the sum, except the last one, vanish, and the present value is

$$
P V_{0}=\frac{1}{r}\left[C-C(1+r)^{-n}\right]=C a_{\bar{n} r}
$$

in accordance with Equation (10).

- Serial bonds is now a general concept in financial markets, characterizing bonds that provide principal repayments over a period of time $!^{6}$ For the special type of a serial bond with a fixed coupon rate $c$ and equal principal repayments over the lifetime $T$, i.e. $\left.Z_{t}=\frac{1}{n}\right]^{7}$ we have only two non-zero terms in the last sum:

$$
P V_{0}=\frac{1}{r}\left[c\left(1-\frac{1}{n} a_{\bar{n} r}\right)+\frac{1}{n}\left(1-(1+r)^{-n}\right)\right]=\frac{c}{r}+\left(1-\frac{c}{r}\right) \frac{1}{n} a_{\bar{n} r} .
$$

The latter expression is Makeham's formula for this particular type of bond.

\section{Applications of FDP and SDP to Less Familiar Present Value Rules}

Indeed, the pricing formulas in Section 3 are well known. We now proceed to a derivation of some more interesting cases along the lines of Proposition 1 and Proposition 2 .

\subsection{Payment streams with constant absolute growth}

Having derived the annuity formula in our setting in Section 3, we now derive the present value of a payment stream with a linear trend, exhibiting constant absolute growth, in the

\footnotetext{
${ }^{6}$ For more information on serial bonds, see, e.g., https://en.wikipedia.org/wiki/Serial_bond or http://www. investopedia.com/terms/s/serialbond.asp.

${ }^{\top}$ As one example of such bonds with a longer history, Danish mortgage bonds as well as Danish government bonds have - with varying intensity over the years - been issued in accordance with this scheme.
} 
same manner. We do so as an introduction to a more general treatment of payment streams where the individual payments $C_{t}$ are given by a polynomial of degree $n$ in time, of which the annuity is the case $n=0$. The case of a polynomial of order $n=1$ is a payment stream composed of an annuity with payment $a_{0}$ and a linear trend with slope $a_{1}$ :

$$
C_{t}=a_{0}+a_{1} t, \quad t=1,2, \ldots, T \text {. }
$$

The present value is the sum of these two ingredients valued individually. The constant term is in itself an annuity. As for the linear trend, its first difference is also an annuity with payment $a_{1}: \Delta_{t}=a_{1} t-a_{1}(t-1)=a_{1}$ for $t=1,2, \ldots, T-1$, whereas the last term is $\Delta_{T}=-a_{1} T$. Using second order differences we have

$$
\Delta_{t}^{2}=0 \text { for } t=1,2, \ldots, T-2, \Delta_{T-1}^{2}=-a_{1}(T+1), \Delta_{T}^{2}=a_{1} T .
$$

Plugging these into Equation (7) we have:

$$
\begin{aligned}
V\left(a_{1} t ; r, T\right) & =\frac{1}{r^{2}}\left[a_{1}(1+r)-a_{1}(T+1)(1+r)^{-(T-1)}+a_{1} T(1+r)^{-T}\right] \\
& =\frac{a_{1}}{r^{2}}\left[1+r-(T+1)(1+r)^{-(T-1)}+T(1+r)^{-T}\right] \\
& =a_{1} \frac{1+r}{r} a_{\bar{T} \mid r}-\frac{a_{1}}{r} T(1+r)^{-T}
\end{aligned}
$$

with limiting value as $T \rightarrow \infty$ :

$$
V\left(a_{1} t ; r, \infty\right)=a_{1} \frac{1+r}{r^{2}}
$$

\subsection{Polynomial payment streams}

We now proceed to show how the difference properties can be used to calculate the present value for the general class of payment streams that have polynomial growth, of which the annuity and the constant absolute growth is a simple example.

Definition 3. A polynomial payment stream is given by the following parametrization for constant coefficients $a_{j}$ :

$$
C_{t}=\sum_{p=0}^{n} a_{p} t^{p}, \quad t=1,2, \ldots, T .
$$


This particular parametrization has the property that the functional form is invariant to taking differences, which we now show. The necessary mathematical prerequisite in order to do so is the binomial theorem, which states that integer powers of the sum of two numbers, $x$ and $y$, can be expressed as the following series expansion: 8

$$
(x+y)^{n}=\sum_{j=0}^{n}\left(\begin{array}{l}
n \\
j
\end{array}\right) x^{j} y^{n-j}
$$

where $\left(\begin{array}{c}n \\ j\end{array}\right)$ is the binomial coefficient:

$$
\left(\begin{array}{l}
n \\
j
\end{array}\right)=\frac{n !}{j !(n-j) !}
$$

Theorem 1. For a polynomial payment stream the following expression holds for an infinite horizon, $T=\infty$, and for $n=0,1,2, \ldots$ :

$$
\begin{aligned}
V\left(t^{n} ; r, \infty\right)=\sum_{t=1}^{\infty} t^{n}(1+r)^{-t} & =\frac{1}{r}\left[1+\sum_{p=0}^{n-1}\left(\begin{array}{l}
n \\
p
\end{array}\right)\left(\sum_{t=1}^{\infty} t^{p}(1+r)^{-t}\right)\right] \\
& =\frac{1}{r}\left[1+\sum_{p=0}^{n-1}\left(\begin{array}{l}
n \\
p
\end{array}\right) V\left(t^{p} ; r, \infty\right)\right]
\end{aligned}
$$

For a finite horizon $T$, the following expression holds for $n=0,1,2, \ldots$ :

$$
V\left(t^{n} ; r, T\right)=\frac{1}{r}\left[1+\sum_{p=0}^{n-1}\left(\begin{array}{l}
n \\
p
\end{array}\right) V\left(t^{p} ; r, T-1\right)-T^{n}(1+r)^{-T}\right] .
$$

Proof. The proof applies the results stated in Proposition 1. See Appendix A.2 for the details.

For a given $n$, the right hand sides of Equations 35 and 36 only contain valuation terms up to order $n-1$. Hence, these formulas are truly recursive.

${ }^{8}$ This is a standard theorem found in mathematical handbooks and in numerous books on mathematical analysis. See, e.g., Section 3.1 of Abramowitz and Stegun (1984). For an overall introduction, including interesting historical references, see, e.g., http://en.wikipedia.org/wiki/Binomial_theorem. 
We have already found this explicit present value formula for the case $n=0$ and $n=1$. We now show how it looks for $n=2, n=3$ and $n=4$. For $n=2$ we have:

$$
\begin{aligned}
& V\left(t^{2} ; r, \infty\right)=\frac{1}{r}\left[1+\frac{1}{r}+2 \frac{1+r}{r^{2}}\right]=\frac{(1+r)(2+r)}{r^{3}}=\frac{r^{2}+3 r+2}{r^{3}} \\
& V\left(t^{2} ; r, T\right)=\frac{(1+r)(2+r)}{r^{2}} a_{\overline{T \mid r}}-(1+r)^{-T} T \frac{r T+2(1+r)}{r^{2}} . \\
& \Delta_{j}^{2}= \begin{cases}2 & \text { for } j=1,2, \ldots, t-2 \\
1-2 t-t^{2} & \text { for } j=t-1 \\
t^{2} & \text { for } j=t\end{cases} \\
& V\left(t^{2} ; r, \infty\right)=\frac{1}{r}\left[1+\frac{1}{r}+2 \frac{1+r}{r^{2}}\right]=\frac{(1+r)(2+r)}{r^{3}}=\frac{r^{2}+3 r+2}{r^{3}} \\
& r V\left(t^{2} ; r, T\right)=1+\frac{3}{r}+\frac{1}{r}\left[2 a_{\bar{t} \mid r}-2(1+r)^{-t}(2+r)+\right] \\
& (1+r)^{-(t-1)}\left(1-2 t-t^{2}\right)+(1+r)^{-t} t^{2} \\
& =1+\frac{3}{r}+\frac{2}{r}\left[a_{\bar{t} \mid r}-(1+r)^{-t}(2+r)\right]+ \\
& \frac{1}{r}(1+r)^{-t}\left(1+r-2 t(1+r)-r t^{2}\right) \\
& r^{2} V\left(t^{2} ; r, T\right)=r+3+2\left[a_{\bar{t} \mid r}+\left(r a_{\bar{t} \mid r}-1\right)(2+r)\right]+ \\
& \left(1-r a_{\bar{t} \mid r}\right)\left((1+r)-2 t(1+r)-r t^{2}\right)
\end{aligned}
$$

For $n=3$ we have:

$$
\begin{aligned}
V\left(t^{3} ; r, \infty\right)= & \frac{1}{r}\left[1+\frac{1}{r}+3 \frac{1+r}{r^{2}}+3 \frac{(1+r)(2+r)}{r^{3}}\right]=\frac{(1+r)\left(r^{2}+6(1+r)\right)}{r^{4}} \\
= & \frac{r^{3}+7 r^{2}+12 r+6}{r^{4}} \\
V\left(t^{3} ; r, T\right)= & \frac{r^{3}+7 r^{2}+12 r+6}{r^{3}} a_{\overline{3} \mid r}- \\
& (1+r)^{-T} T\left[\frac{r^{2} T^{2}+3 \operatorname{Tr}(1+r)+3(1+r)(2+r)}{r^{3}}\right] .
\end{aligned}
$$


For $n=4$ we have:

$$
\begin{gathered}
V\left(t^{4} ; r, \infty\right)=\frac{1}{r}\left[1+\frac{1}{r}+4 \frac{1+r}{r^{2}}+6 \frac{(1+r)(2+r)}{r^{3}}+4 \frac{r^{3}+7 r^{2}+12 r+6}{r^{4}}\right] \\
=\frac{1}{r^{5}}\left[r^{4}+15 r^{3}+48 r^{2}+60 r+24\right] \\
V\left(t^{4} ; r, T\right)=\frac{1}{r^{4}}\left[r^{4}+15 r^{3}+48 r^{2}+60 r+24\right] a_{\overline{4} \mid r}-(1+r)^{-T} T \cdot \\
\frac{T^{3} r^{3}+4 T^{2} r^{2}(1+r)+6 \operatorname{Tr}(1+r)(2+r)+4(1+r)\left(r^{2}+6(1+r)\right)}{r^{4}} .
\end{gathered}
$$

These higher order powers are naturally found in the calculation of the derived risk measures duration and convexity, to which we turn in Sections 5 and 6 .

\subsection{Present value and yield after tax}

In this section we apply the FDP to derive a relationship between the present value before tax and the present value after tax in order to find and interpret the yield after tax for a taxable investor. We assume that the payments on a fixed rate bond are taxed with the tax rate $\tau$ and that capital gains are taxed upon realization with the rate $\tau_{c}$. Furthermore, we assume that the bond has been sold at a discount. Tax laws and practices in many countries apply the logic of taxing current interest payments as they occur, but only tax capital gains and losses when actual repayments - or sales transactions - take place. Furthermore, the tax on capital gains is often at a lower rate than the tax rate applied to interest payments.

In this setting we consider a bond held until maturity and apply the results obtained in Example 4 above. On an after tax basis this gives rise to the following payment pattern:

$$
\begin{gathered}
C_{t}=c(1-\tau)\left(\sum_{j=t}^{n} Z_{j}\right)+Z_{t}\left(1-(1-V(\underline{C} ; r, n)) \tau_{c}\right) \\
C_{t+1}=c(1-\tau)\left(\sum_{j=t+1}^{n} Z_{j}\right)+Z_{t+1}\left(1-(1-V(\underline{C} ; r, n)) \tau_{c}\right) \\
C_{t+1}-C_{t}=\left(Z_{t+1}-Z_{t}\right)\left(1-(1-V(\underline{C} ; r, n)) \tau_{c}\right)-c(1-\tau) Z_{t} .
\end{gathered}
$$

Finding the yield after tax, which we denote as $\widetilde{r}$, requires that we find the relevant discount rate and discount factor that, when applied to the sequence of payments after tax, gives the 
present value $V(\underline{C} ; r, n)$. There is no analytic solution available for this, but we can at least get expressions that enable an interpretation of the forces at work.

Applying the FDP, as expressed in 24, to the sequence of after tax payments means we are looking for a solution to the equation:

$$
\begin{aligned}
V(\underline{C} ; r, n)=\frac{1}{\widetilde{r}}\left[c(1-\tau)\left(1-\sum_{t=1}^{n} Z_{t}(1+\widetilde{r})^{-t}\right)+Z_{1}\left(1-\tau_{c}+\tau_{c} V(\underline{C} ; r, n)\right)+\right. \\
\\
\left.\sum_{t=1}^{n}\left(Z_{t+1}-Z_{t}\right)\left(1-\tau_{c}+\tau_{c} V(\underline{C} ; r, n)\right)(1+\widetilde{r})^{-t}\right] .
\end{aligned}
$$

In terms of the return after tax, $\widetilde{r}$, applied to the actual investment $V(\underline{C} ; r, n)$, we can rewrite Equation (53) by using the FDP property in reverse as:

$$
\widetilde{r} V(\underline{C} ; r, n)=c(1-\tau)\left(1-\sum_{t=1}^{n} Z_{t}(1+\widetilde{r})^{-t}\right)+\widetilde{r}\left(1-\tau_{c}+\tau_{c} V(\underline{C} ; r, n)\right) \sum_{t=1}^{n} Z_{t}(1+\widetilde{r})^{-t}
$$

In this expression we recognize $\left(1-\tau_{c}+\tau_{c} V(\underline{C} ; r, n)\right) \sum_{t=1}^{n} Z_{t}(1+\widetilde{r})^{-t}$ as the present value of after tax capital gains. As for the first term we need to rewrite the expression as shown in Equation (55):

$$
\begin{aligned}
& c(1-\tau) \sum_{t=1}^{n} Z_{t}\left(1-(1+\widetilde{r})^{-t}\right)=\widetilde{r} c(1-\tau) \sum_{t=1}^{n} Z_{t} \frac{1-(1+\widetilde{r})^{-t}}{\widetilde{r}}= \\
& \widetilde{r} c(1-\tau) \sum_{t=1}^{n} Z_{t} \sum_{j=1}^{t}(1+\widetilde{r})^{-j}=\widetilde{r} \sum_{j=1}^{n}(1+\widetilde{r})^{-j}\left[c(1-\tau) \sum_{t=j}^{n} Z_{t}\right] .
\end{aligned}
$$

This shows that the first term in Equation (54) is the after tax return $(\widetilde{r})$ of the present value of coupon payments after tax, which is the last term in parenthesis in Equation (55). There are two forces at work here. One is the value of the deferral of capital gains taxation. The other is the preferential treatment of capital gains whenever $\tau_{c}<\tau$. The latter effect is absent when the tax rates applied are identical, but the deferral effect is present in all cases. We limit ourselves to a consideration of bullet bonds and add the suffix $n$ to the unknown yield after tax for the bond with time to maturity $n$. For such bonds, Equation (53) can be 
rewritten as:

$$
\begin{aligned}
\frac{c}{r}+\left(1-\frac{c}{r}\right)(1+r)^{-n}= & c(1-\tau) \sum_{t=1}^{n}\left(1+\widetilde{r}_{n}\right)^{-t}+ \\
& \left(1-\tau_{c}\left(1-\frac{c}{r}\right)\left(1-(1+r)^{-n}\right)\right)\left(1+\widetilde{r}_{n}\right)^{-n}
\end{aligned}
$$

Except for $n=1$, there is no analytic solution available for this equation. However, some qualitative properties, concerning the effect of the maturity and the possible lower taxation of capital gains at the rate $\tau_{c}<\tau$, can be shown:

a) As $n \rightarrow \infty$ the left hand side becomes $\frac{c}{r}$, whereas the right hand side becomes $\frac{c(1-\tau)}{\widetilde{r}_{n}}$. Hence, $\lim _{n \rightarrow \infty} \widetilde{r}_{n}=r(1-\tau)$; and $\widetilde{r}_{n} \simeq r(1-\tau)$ for bullet bonds with very long maturity. $\tau_{c}$ becomes more and more irrelevant as $n \rightarrow \infty$, because the present value of the tax burden on capital gains vanishes in the limit.

b) For the very short term bond, $n=1$, we can solve explicitly for $\widetilde{r}_{1}$ :

$$
\begin{aligned}
\frac{c}{r}+\left(1-\frac{c}{r}\right)(1+r)^{-1} & =c(1-\tau)\left(1+\widetilde{r}_{1}\right)^{-1}+\left(1-\tau_{c}\left(1-\frac{c}{r}\right) \frac{r}{1+r}\right)\left(1+\widetilde{r}_{1}\right)^{-1} \Rightarrow \\
\widetilde{r}_{1} & =\frac{c(1-\tau)}{V(1+c ; r, 1)}+\left(\frac{1}{V(1+c ; r, 1)}-1\right)\left(1-\tau_{c}\right) \\
& =c(1-\tau) \frac{1+r}{1+c}+\left(\frac{r-c}{1+c}\right)\left(1-\tau_{c}\right) .
\end{aligned}
$$

The first term is the part of the return that is due to the coupon payment. The second part is the return due to the capital gain. Consider the two special cases, $\tau_{c}=0$ and $\tau_{c}=\tau$, respectively.

When $\tau_{c}=\tau$ there is neither a differential tax treatment of the two sources of income nor is there an effect from deferring capital gains. Hence, $\widetilde{r}=r(1-\tau)$. When $\tau_{c}=0$ we have the advantage of tax free capital gains as reflected in the last term in Equation (58):

$$
\widetilde{r}=r(1-\tau)+\left(\frac{1}{V(1+c ; r, 1)}-1\right) \tau=r(1-\tau)+\frac{(r-c) \tau}{1+c} .
$$

c) When $\tau_{c}=0$ the after tax yield is a decreasing function of the maturity $n$. The effect of the tax free capital gain decreases as its fraction of the present value of the payment stream decreases 9

\footnotetext{
${ }^{9}$ Mathematical proofs of the claims in (c) and (d) are outside the main scope of this paper, but are
} 


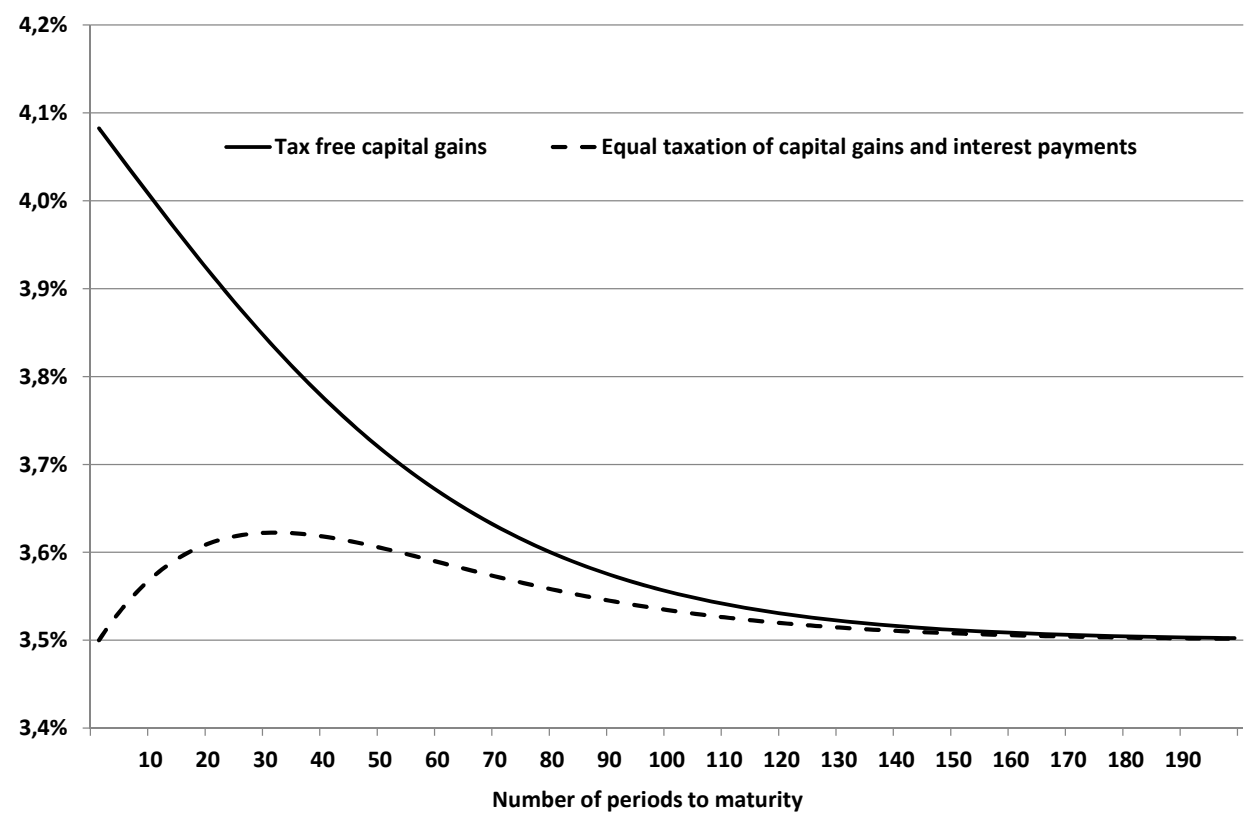

Figure 1: Yield after tax for bullet bond, $c=3 \%, r=5 \%, \tau=30 \%$, as a function of time to maturity.

d) When $\tau_{c}=\tau$ there is only the deferral effect. The after tax yield is a hump-shaped function of the maturity $n$, reflecting two counteracting forces at work. Although the capital gains component increases in present value terms with the maturity $n$, the present value of any given amount of capital gain decreases due to discounting. The yield is an annualized measure that takes the latter effect into account 10

In Figure 1 we graphically show the result for $\tau_{c}=0$ and $\tau_{c}=\tau$, respectively, for $c=3 \%$, $r=5 \%$ and $\tau=30 \%$. For tax-free capital gains, the effect on the after tax yield with identical taxation of interest payments and capital gains (3.5\%) is significant for also longer maturities as shown by the solid curve. As for the tax deferral effect in and of itself, which is shown by the dashed line, it has a rather small impact on the yield after tax.

available upon request.

${ }^{10}$ Bond investment analyses in the same spirit are found in Roll (1984) and Rodriguez (1988). 


\subsection{Cyclically varying payment streams}

Cyclical variation in payment streams is common for producers of agricultural products which are sensitive to seasonal changes. Examples of extensive empirical analyses are found in, e.g., Sørensen (2002) and Geman and Nguyen (2005), both of which established a framework of analysis for seasonal variations in agricultural commodity prices and documented how available inventories played a significant role in the dynamics of these prices. Cyclical variation in payment streams as well as in physical magnitudes is also a widespread phenomenon in the energy sector. The following examples 6 and 7 describe two such cases involving natural gas.

Cyclical patterns are also well documented for broad measures of economic production, and firms whose cash flows are especially sensitive to such patterns are commonly referred to as cyclical. Predictable seasonal patterns also occur for broad categories of retail sales which are due to the coincidence of certain holidays, such as Christmas, Hanukkah, and Kwanzaa. More generally, the fourth quarter of each year is widely regarded as an essential period for large categories of retail sales.

Yet, despite this common perception, formal present value calculations typically fail to address the seasonal component of sales and for the corresponding cash flows generated by retail sales as well as by agricultural products.

Example 5: Cash flows with alternating signs

To illustrate the general issue, consider a perpetual payment stream of constant magnitude, $a$, but alternating signs, $\underline{C}=\{a,-a, a,-a, \ldots, a,-a\}$. For any such cash flow, the pattern of second differences is proportional to the cash flow itself with multiplicative factor 4 :

$$
\Delta=\{-2 a, 2 a,-2 a, 2 a, \ldots\}=(-2) \underline{C}, \quad \Delta^{2}=4 \underline{C} .
$$

Hence, by Proposition 2 we immediately have that:

$$
\begin{gathered}
r V\left((-a)^{t-1} ; r, \infty\right)=a-\frac{2 a}{r}+\frac{4}{r} V\left((-a)^{t-1} ; r, \infty\right) \Rightarrow \\
\left(r^{2}-4\right) V\left((-a)^{t-1} ; r, \infty\right)=a(r-2) \Rightarrow V\left((-a)^{t-1} ; r, \infty\right)=\frac{a}{2+r} .
\end{gathered}
$$

The finite horizon case is the difference between the immediately starting and the forward starting payment stream. Here it matters whether the number of payments is even or odd. 
Assuming that $C_{1}=a$ we have that:

$$
V\left((-a)^{t-1} ; r, T\right)= \begin{cases}a \frac{1-(1+r)^{-T}}{2+r} & \text { for } T \text { even } \\ a \frac{1+(1+r)^{-T}}{2+r} & \text { for } T \text { odd }\end{cases}
$$

A simple generalization of this is the case where

$$
\underline{C}=\{a,-b, a,-b, \ldots, a,-b, \ldots\}
$$

which is a combination of an annuity with value $\frac{a-b}{2}$ and the cyclical cash flow $\pm \frac{a+b}{2}$. Hence, the present value is:

$$
V(\underline{C} ; r, T)= \begin{cases}\frac{a-b}{2} a_{\bar{T} \mid r}+\frac{a+b}{2} \frac{1-(1+r)^{-T}}{2+r}=\frac{a(1+r)-b}{(2+r) r}\left(1-(1+r)^{-T}\right) & \text { for } T \text { even } \\ \frac{a-b}{2} a_{\bar{T} \mid r}+\frac{a+b}{2} \frac{1+(1+r)^{-T}}{2+r}=\frac{a(1+r)-b+(b(1+r)-a)(1+r)^{-T}}{(2+r) r} & \text { for } T \text { odd } \\ \frac{a(1+r)-b}{(2+r) r} & \text { for } T=\infty\end{cases}
$$

Such a cash flow reflects the situation for, e.g., a skiing resort where the income generated is confined to the winter season, but where the summer season involves maintenance costs. Similar patterns are known for amusement parks, camping grounds etc.

Even the simple example of alternating signs, cf. Equations (59) - 61, highlights the potential significance of the general issue. For example, for a projected cash flow that is presumed to exhibit cyclical variation, one might reasonably presume that $a$ is positive at the beginning of a cycle that is deemed to be favorable for that cash flow but negative at the beginning of a cycle that is deemed to be unfavorable for that cash flow. If the magnitude of the early effect, $a$, is relatively large compared to the rate of discount, r, Equation (60) establishes that the effect on valuation is roughly one half the magnitude of the variation effect, $a$. For example, one common approach considers the current market price for a stock in relation to various measures such as: a) the average value of a given number of prior earnings; or b) an estimate of average future earnings; or c) an average rate of historical growth in cash flow. None of these common approaches consider the effect of the current status of the cyclical variation per se. Yet ignoring this fundamental effect could lead to potentially significant errors for valuations.

Addressing valuation issues that arise for cyclical variation in payment streams also highlights the need for extended discussions of the effects of risk on valuations. Traditional discussions 

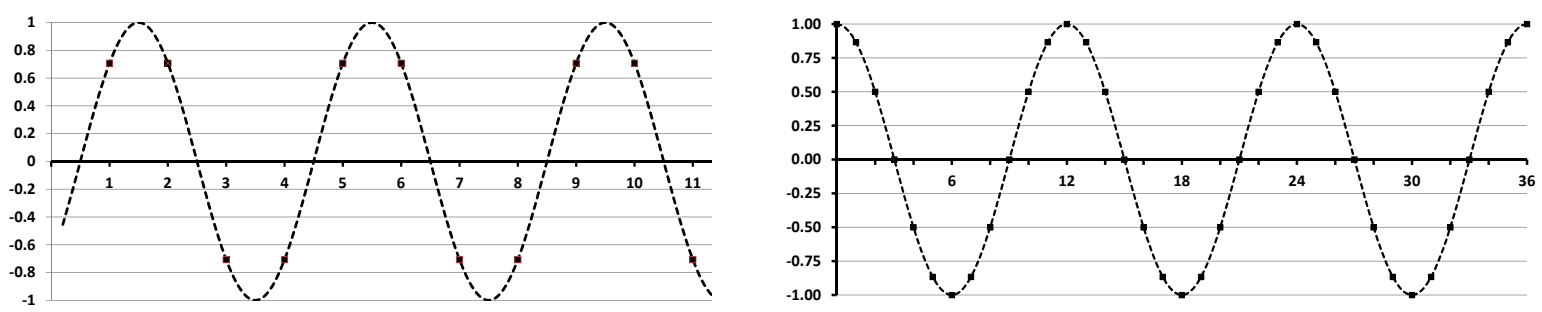

Figure 2: Cyclical cash flows. Left panel quarterly payments with $\omega=\pi / 2$ and $\phi=-\pi / 4$. Right panel monthly payments with $\omega=\pi / 6$ and $\phi=\pi / 3$.

of risk focus on two potential indirect effects. First, required rates of return can increase or decrease if the assumed extent of risk increases or decreases. And second, required rates of return can increase or decrease if the price per unit of risk increases or decreases. As illustrated for the simple case of cyclical variation considered in Example 5, and as will be expanded upon in the discussion of Example 8, the analysis of cyclical variation identifies two direct effects of deviations from traditional projections of cash flows. First, there is a direct effect of the magnitude of the variation, as measured by the parameter $a$. And second, the stage of the cycle, as reflected by the sign of $a$, has a direct effect on the present value of a cash flow. Despite such general acknowledgements, textbooks that focus on asset valuations rarely, if ever, include any formal discussion of how cyclical variation impacts the present value of a cash flow.

In order to proceed to a more general result we assume that cyclical variations can be described by a sine function, which may be variations around a certain level or around a moving trend:

$$
C_{t}=\sin (\omega t+\phi), t=1,2, \ldots, T
$$

Cyclically varying payment streams have frequencies $\omega=2 \pi / h$ for some integer $h$, and thus repeat themselves after $h$ payments. The naturally occurring frequencies are $h=2$ (semiannually), $h=4$ (quarterly) and $h=12$ (monthly). Figure 2 shows the cases with quarterly payments $(h=4, \omega=\pi / 2)$ and monthly payments $(h=12, \omega=\pi / 6)$.

Real life examples of cyclical payment streams often appear as derived consequences embedded in contracts rather than being directly expressed as contract terms. Before presenting our general analytic results for cyclically varying payment streams in Theorem 2, we present two such real life examples. 


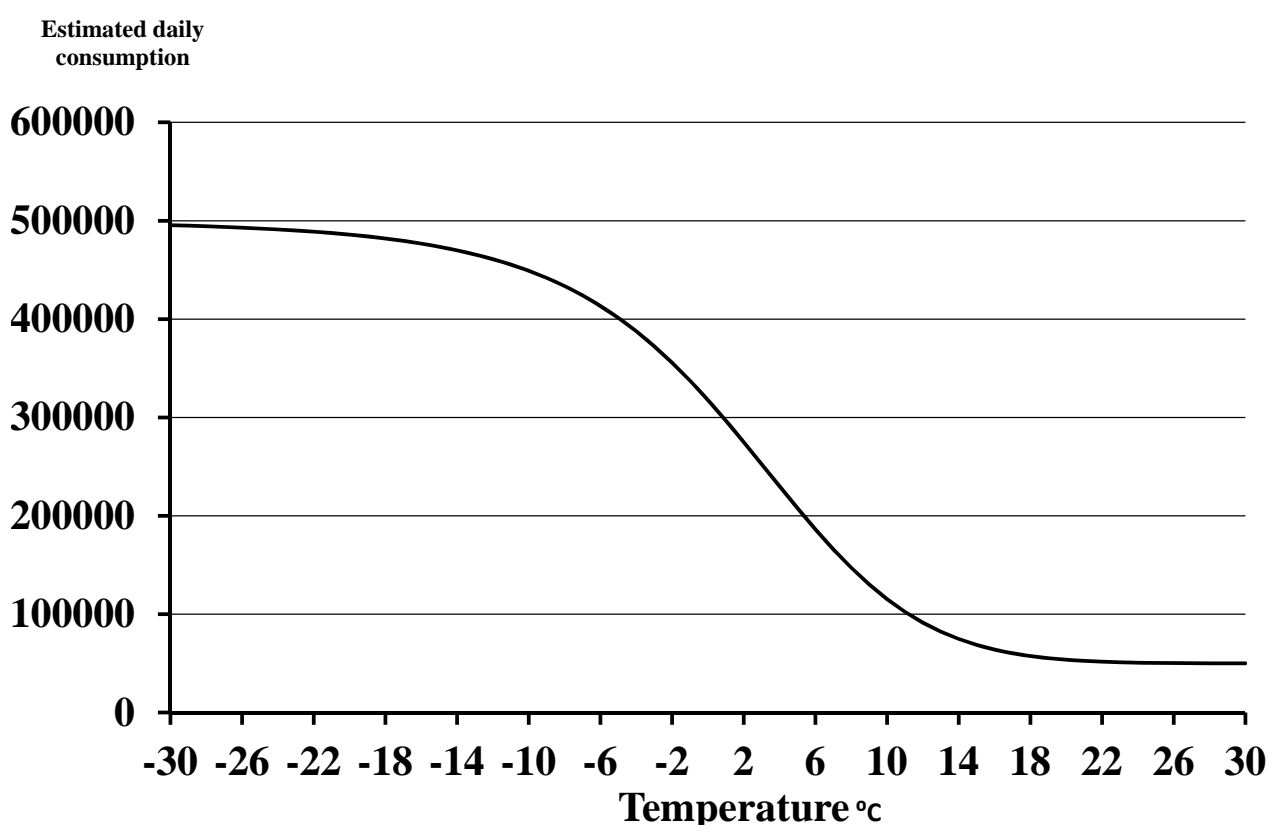

Figure 3: Example of the Sigmoid function

Example 6: Calculating your gas bill

The market for natural gas is cyclical in the sense that both the volume sold and the price varies with the heating seasons. This has given rise to a number of special arrangements, both in the terms of financial contracts and in terms of contracts for physical facilities such as storage capacity.

Customers are usually only reporting their cumulative consumption at longer intervals. An often used feature designed to capture the variation in production costs and pass this variation on to consumers is the sigmoid function that calculates an imputed consumption per calendar unit. Afterwards, this imputed consumption is multiplied with the spot price for that same calendar unit. An example of the sigmoid function is given by the following formula:11

$$
C V \cdot\left(\frac{4.5}{1+\left(\frac{38}{40-T}\right)^{7.5}}+0.5\right)
$$

where $C V$ is a scaling factor adjusted to the individual consumer's consumption and $T$ is

\footnotetext{
${ }^{11}$ The concrete case shown here is a contract used by the gas company WINGAS for gas distribution in the network Net Connect Germany ("NCG"). We are grateful to senior manager Peter Lyk-Jensen from the Danish Oil and Natural Gas Company (DONG) for providing us with contracts and background knowledge concerning the market for gas.
} 
the average temperature for the delivery day. The upper and lower limits of the function in parenthesis are $4.5+0.5=5$ and 0.5 , respectively. Figure 3 shows this function for $C V=100.000$.

It is well documented that gas prices vary cyclically over the year, significantly correlated with temperature variations. Hence, the product of the gas price and the estimated daily consumption shows a cyclical pattern, being the product of two positively correlated cyclically varying measures.

Variants of this type of contract use, e.g., monthly arrangements where the gas price is an average of one month ahead forward prices. Gas may also be sold on fixed price contracts; in such cases the gas company is carrying some or all of the risk due to cyclical variations and is left with a need to find hedge instruments to deal with this.

Example 7: Contracts for storage facilities

Contracts for storage facilities for natural gas or means for the production of electricity are common ${ }^{12}$ Such storage facilities enable producers to exploit, e.g., the cyclical variation in gas prices by buying up gas for storage during the summer season and extract gas from such inventories during the winter season.

One type of contract is a fixed price contract which - in addition to paying for operational costs - gives the buyer the option to inject, store and later extract a certain amount of gas. In this way the buyer has an option on the variation of the seasonal spread of gas prices. Another type of contract have payments that are indexed to gas prices such that the buyer and the storage operator shares the potential gains from the variations of the seasonal spread. Under all circumstances there are upper physical limits as to the capacity with which injections and extractions can take place. But given the option feature of such contracts the storage operator may also include swing option features into the contract specifying a minimum amount of storage of capacity to be used.

The following Theorem 2 states the results for the present value of a cyclically varying payment stream.

Theorem 2. For a cyclically varying payment stream with period $\omega=2 \pi / h$, as defined in

\footnotetext{
${ }^{12}$ Sometimes referred to as SBUs (Standard Bundled Products). A broader description of such contracts can be found at homepages of companies supplying such contracts and facilities. See, e.g., http://www. centrica-sl.co.uk and https://www.storengy.com/en
} 
Equation (64), the following relationships hold:

$$
\begin{aligned}
& V(\underline{C} ; r, T)=\sum_{t=1}^{T} \sin (\omega t+\phi)(1+r)^{-t}= \\
& \frac{(1+r) \sin (\omega+\phi)-\sin (\phi)-(1+r)^{-T}((1+r) \sin (\omega(T+1)+\phi)-\sin (\omega T+\phi))}{1+(1+r)^{2}-2(1+r) \cos (\omega)} \\
& V(\underline{C} ; r, \infty)=\frac{(1+r) \sin (\omega+\phi)-\sin (\phi)}{1+(1+r)^{2}-2(1+r) \cos (\omega)} .
\end{aligned}
$$

When $T$ represents a number of full cycles in the sense that $\omega T=n(2 \pi)$ for some integer $n$, the expression for the present value in (66) is simplified to:

$$
V(\underline{C} ; r, T)=\left(1-(1+r)^{-T}\right) \frac{(1+r) \sin (\omega+\phi)-\sin (\phi)}{1+(1+r)^{2}-2(1+r) \cos (\omega)} .
$$

Proof. For the proof of $(66)$, see Appendix A.3. The result in $(68)$ is merely a restatement of the result in Equation $(66)$ for full cycles, where $\sin (\omega T+\phi)=\sin (\phi)$ and $\sin (\omega(T+1)+\phi)=$ $\sin (\omega+\phi)$.

Example 8: Some cases of cyclically varying payment streams

The case with $h=2$ was shown in Example 5. This corresponds to $\omega=\pi$ and $\cos (\omega)=-1$. The payments are alternating in sign with the same numerical value, depending on the value of the phase $\phi$ :

$$
\sin (\pi+\phi), \sin (\phi)=-\sin (\pi+\phi), \sin (\pi+\phi),-\sin (\pi+\phi), \ldots
$$

For the sequence $(1,-1,1,-1, \ldots)$ we have $\phi=-\frac{\pi}{2}$ and $\sin (\phi)=-1$.

The quarterly varying sequence with $h=4$ and $\omega=\pi / 2$ produces the payments

$$
\sin \left(\frac{\pi}{2}+\phi\right), \sin (\pi+\phi), \sin \left(\frac{3}{2} \pi+\phi\right), \sin (\phi), \sin \left(\frac{\pi}{2}+\phi\right), \ldots
$$

When $\phi=0$ this is simply $1,0,-1,0,1, \ldots$ When $T$ is a multiple of 4 , i.e. a number of full "cycles", we have for $\phi=0$ the present value:

$$
\sum_{t=1}^{T} \sin \left(\frac{\pi}{2} t\right)(1+r)^{-t}=\frac{(1+r)\left(1-(1+r)^{-T}\right)}{1+(1+r)^{2}}=a_{\bar{T} r}\left[r \frac{1+r}{1+(1+r)^{2}}\right]
$$


where $r \frac{1+r}{1+(1+r)^{2}}$ is the equivalent annual annuity ${ }^{13}$ for the infinite sequence with present value equal to $\frac{1+r}{1+(1+r)^{2}}$.

Setting $\phi=-\pi / 4$ gives the sequence $1,1,-1,-1,1,1,-1,-1, \ldots$, scaled by $\sin (\pi / 4)=1 / \sqrt{2}$, which is depicted in the left panel in Figure 2, Such a sequence corresponds to a payment stream that varies with heating costs, e.g., in a yearly cycle starting with "autumn, winter" followed by "spring, summer". The present value is:

$$
\begin{aligned}
& \sum_{t=1}^{T} \sin \left(\frac{\pi}{2} t-\frac{\pi}{4}\right)(1+r)^{-t}= \\
& \frac{(1+r) \sin \left(\frac{\pi}{4}\right)-\sin \left(-\frac{\pi}{4}\right)-(1+r)^{-T}\left((1+r) \sin \left(\frac{\pi}{2} T+\frac{\pi}{4}\right)-\sin \left(\frac{\pi}{2} T-\frac{\pi}{4}\right)\right)}{1+(1+r)^{2}}
\end{aligned}
$$

which for a full yearly cycle, with $T$ being a multiple of 4 , becomes

$$
\begin{aligned}
& \sum_{t=1}^{T} \sin \left(\frac{\pi}{2} t-\frac{\pi}{4}\right)(1+r)^{-t}= \\
& \frac{(2+r)}{1+(1+r)^{2}}\left[1-(1+r)^{-T}\right] \sin \left(\frac{\pi}{4}\right)=\frac{(2+r)}{1+(1+r)^{2}}\left[1-(1+r)^{-T}\right] 0.7071 .
\end{aligned}
$$

These considerations can - as one variant on a theme - be carried over to payment streams that exhibit cyclical variations around a constant or growing trend, either with variations of constant amplitude or variations with amplitudes that grow with the underlying trend. In Section 5 we show a numerical example of such a payment stream.

\section{Duration}

The usual definition of the duration highlights its role as a measure of the elasticity of the present value with respect to the discount factor $1+r$. More precisely, the traditional

\footnotetext{
${ }^{13}$ Also known as the equivalent annual cost (EAC).
} 
Macaulay/Redington duration measure is ${ }^{14}$

$$
D=-\frac{\partial V(\underline{C} ; r)}{\partial(1+r)} \frac{(1+r)}{V(\underline{C} ; r)}=\frac{\sum_{t=1}^{T} C_{t} t(1+r)^{-t}}{\sum_{t=1}^{T} C_{t}(1+r)^{-t}}=\sum_{t=1}^{T} \omega_{t} t
$$

where $\omega_{t}$ is the relative weight of the individual payment $C_{t}$ in the present value of the payment stream: $\omega_{t}=C_{t}(1+r)^{-t} / \sum_{t=1}^{T} C_{t}(1+r)^{-t}$. The factor $1+r$ is typically excluded in the expression referred to as modified duration. However, it is usually included in presentations that focus on "effective maturity" or "immunization horizon" in accordance with the weighted sum of maturities for the individual payments. Except for the term $1+r$, the duration is also the coefficient of the first-order term in the following Taylor series expansion: ${ }^{15}$

$$
V(\underline{C} ; r+\Delta r, T) \simeq V(\underline{C} ; r, T)\left[1-D \frac{\Delta r}{1+r}\right] .
$$

As is apparent from Equation (72), the duration can be calculated without the use of differentiations and summation of series if one can easily calculate both the numerator and the denominator. We now demonstrate the use of this technique to various payment streams.

\subsection{Duration measures for polynomial payment streams}

Having found the magnitudes $V\left(t^{p} ; r, T\right)$ in Theorem 1, the duration can be calculated for any polynomial payment stream with power $p$ as the value weighted sum of these individual power terms:

$$
\begin{aligned}
V\left(\sum_{p=0}^{n} a_{p} t^{p} ; r, T\right) & =\sum_{p=0}^{n} a_{p} V\left(t^{p} ; r, T\right) \\
\frac{V\left(\sum_{p=0}^{n} a_{p} t^{p+1} ; r, T\right)}{V\left(\sum_{p=0}^{n} a_{p} t^{p} ; r, T\right)} & =\sum_{p=0}^{n} W_{p} \frac{V\left(t^{p+1} ; r, T\right)}{V\left(t^{p} ; r, T\right)}
\end{aligned}
$$

where the weights $W_{p}$ are given by $W_{p}=a_{p} V\left(t^{p} ; r, T\right) / V\left(\sum_{p=0}^{n} a_{p} t^{p} ; r, T\right)$.

Example 1](cont.): Duration for an annuity

For the annuity this is simple. We denote this duration measure as $D_{\text {ann }}$ and insert the

\footnotetext{
${ }^{14}$ In this setting we rely on an interpretation provided by Redington $(1952)$, where the discount rate is the yield, rather than the interpretation attributed to Macaulay (1938). There is no assumption on our part of a flat term structure.

${ }^{15}$ The term $1+r$ appears due to the differentiation of the discount factor $(1+r)^{-t}$. In case one uses continuous compounding, this term disappears.
} 
results for $n=0,1$ and $a_{0}=1$ from Equation 36 to arrive at:

$$
D_{a n n .}=\frac{V(t ; r, T)}{V(1 ; r, T)}=\frac{\frac{1+r}{r} a_{\overline{T r}}-\frac{T}{r}(1+r)^{-T}}{a_{\overline{T r} r}}=\frac{1+r}{r}-\frac{T}{(1+r)^{T}-1} .
$$

As $T \rightarrow \infty$, this expression converges to the duration measure for the perpetuity: $(1+r) / r$.

Example 2 (cont.): Duration for payment streams with a constant percentage growth

The duration for a payment stream that exhibits a constant percentage growth can be found in a simple way by using the FDP.

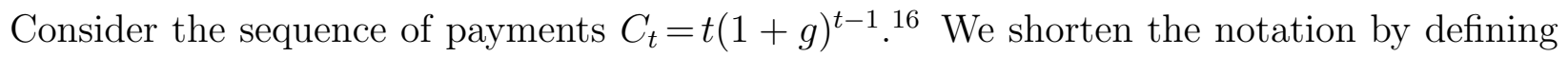
$G_{t} \equiv(1+g)^{t-1}$. The first differences are:

$$
\Delta_{t}=(t+1) G_{t+1}-t G_{t}= \begin{cases}G_{t}(g t+(1+g)) & (\text { for } t \leq T-1) \\ -T G_{T} & (\text { for } t=T)\end{cases}
$$

We now use the basic result from Proposition 1. In order to obtain compatible present value expressions we include the $T$-term with the same functional form as the other terms in the present value of the first differences; to compensate for this, we subtract this extra term explicitly afterwards. By doing so we obtain:

$$
\begin{aligned}
r V\left(t G_{t} ; r, T\right)= & 1+g V\left(t G_{t} ; r, T\right)-g T G_{T}(1+r)^{-T}+ \\
& (1+g) V\left(G_{t} ; r, T\right)-G_{T+1}(1+r)^{-T}-T G_{T}(1+r)^{-T} .
\end{aligned}
$$

After having collected terms this reduces to:

$$
(r-g) V\left(t G_{t} ; r, T\right)=1-\left(\frac{1+r}{1+g}\right)^{-T}-T G_{T+1}(1+r)^{-T}+(1+g) V\left(G_{t} ; r, T\right)
$$

Finally, the expression for the duration is:

$$
D_{\text {growth }}=\frac{V\left(t G_{t} ; r, T\right)}{V\left(G_{t} ; r, T\right)}=1+\frac{1+g}{r-g}-\frac{T}{\left(\frac{1+r}{1+g}\right)^{T}-1}=\frac{1+r}{r-g}-\frac{T}{\left(\frac{1+r}{1+g}\right)^{T}-1} .
$$

The asymptotic limit as $T \rightarrow \infty$ is $(1+r) /(r-g)$.

\footnotetext{
${ }^{16}$ Without loss of generality, we scale the payment stream such that $C_{1}=1$.
} 
Example 3 (cont.): Duration of a bullet bond

The bullet bond can be thought of as a portfolio of zero-coupon bonds, where each cash flow is a separate zero-coupon bond or it can be thought of as a portfolio that contains one of an annuity and one of a zero-coupon bond where the zero-coupon bond's face value is equal to the bullet bond's principal. Viewing the bullet bond as the latter portfolio, we can calculate the duration of the portfolio as the weighted average of the duration of the annuity and the zero-coupon bond, where the weights correspond to their share of the total portfolio value. For the zero-coupon bond, the duration is straightforward to find from the definition, cf. (72), as $T$ - there is only one payment at time $T$ leading to $\omega_{T}=1$. We therefore find the duration of a bullet bond with coupon rate $c, D_{\text {bullet }}$, to be ${ }^{17}$

$$
\begin{aligned}
D_{\text {bullet }} & =\frac{c a_{\overline{T r} r}}{c a_{\bar{T} r}+(1+r)^{-T}} D_{a n n .}+\frac{(1+r)^{-T}}{c a_{\bar{T} r}+(1+r)^{-T}} T \\
& =\frac{c a_{\overline{T r} r}}{c a_{\bar{T} r}+(1+r)^{-T}} \frac{1+r}{r}+\frac{(1+r)^{-T}}{c a_{\overline{T r} r}+(1+r)^{-T}} T\left[1-\frac{c}{r}\right] \\
& =\frac{1+r}{r}+\frac{(1+r)^{-T}}{c a_{\bar{T} r}+(1+r)^{-T}}\left[\frac{T(r-c)-(1+r)}{r}\right] .
\end{aligned}
$$

That is, the duration is a weighted sum of the duration of the perpetual annuity and $T\left[1-\frac{c}{r}\right]$, cf. 82$]$. Furthermore, when a bullet bond is sold at a discount, the duration as a function of time to maturity is no longer monotonic, but will overshoot its asymptotic limit $(1+r) / r$, cf. (83). Although this is guaranteed to happen, it may only happen for times to maturity $T$ beyond what is found in actual financial markets. Additionally, the magnitude of this overshooting is likely to be very small due to the discount factor $(1+r)^{-T}$ in front.

Figure 4 shows these duration measures as a function of time to maturity for the annuity and the bullet bond with $c=4 \%$ and $r=8 \%$.

Example 12: Duration for a payment stream with constant absolute growth

We now reconsider the duration of the payment stream with linear growth from subsection 4.1. We denote its duration by $D_{l i n}$. and use the results from Equations (30) and (38). This

\footnotetext{
${ }^{17}$ The expressions in Equations (81) through 83) were derived in various versions in Chua (1984), Chua (1985) and Babcock (1985). The expression in Equation (82) is often referred to as Babcock's formula. The duration formulas presented here are valid at coupon dates only. Chua (1988) presents a duration formula that is valid at any intermediate date between two coupon dates. His formula is simply the analytical duration at the last coupon date shortened by the fraction of the period that has passed since the last coupon date.
} 


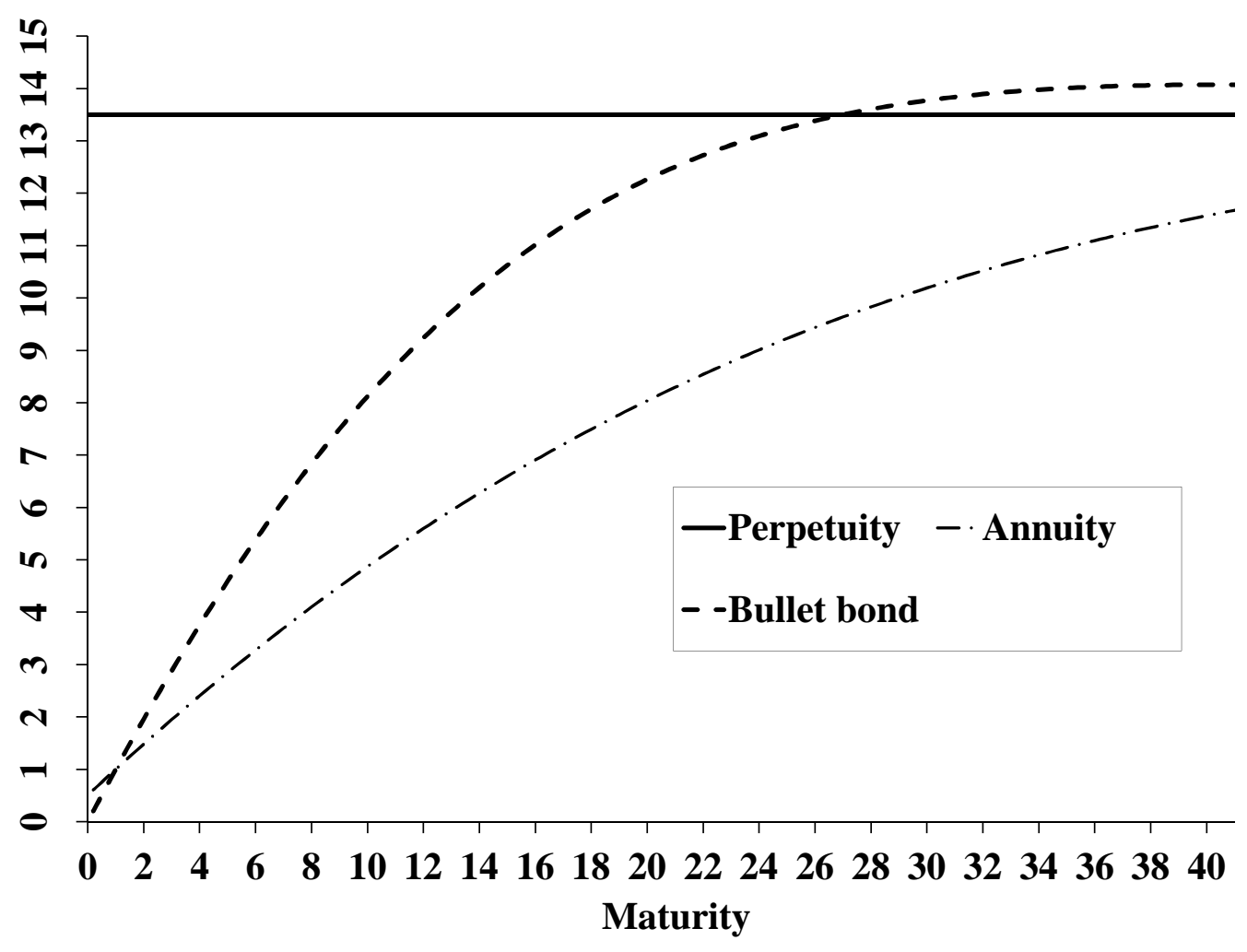

Figure 4: Duration measure as a function of time to maturity for the annuity and the bullet bond with $c=4 \%$ and $r=8 \%$.

shows that:

$$
D_{\text {lin. }}=\frac{V\left(t^{2} ; r, T\right)}{V(t ; r, T)}=\frac{\frac{1}{r^{2}}\left[(1+r)(2+r) a_{\overline{T r}}-(2(1+r)+r T) T(1+r)^{-T}\right]}{\frac{1+r}{r} a_{\overline{T r} r}-\frac{T}{r}(1+r)^{-T}} .
$$

Multiplying through by $r$ and collecting terms, we find the following closed form expression:

$$
D_{\text {lin. }}=\frac{2+r}{r}-\frac{T(T+1)(1+r)^{-T}}{(1+r) a_{\bar{T} \mid r}-T(1+r)^{-T}} .
$$

Figure 5 shows this duration measure as a function of time to maturity for $r=5 \%$.

The duration for the cash flow with linear growth is significantly larger than for the annuity. As $T \rightarrow \infty$, this expression converges to $(2+r) / r$, which is approximately double the value of the annuity. 


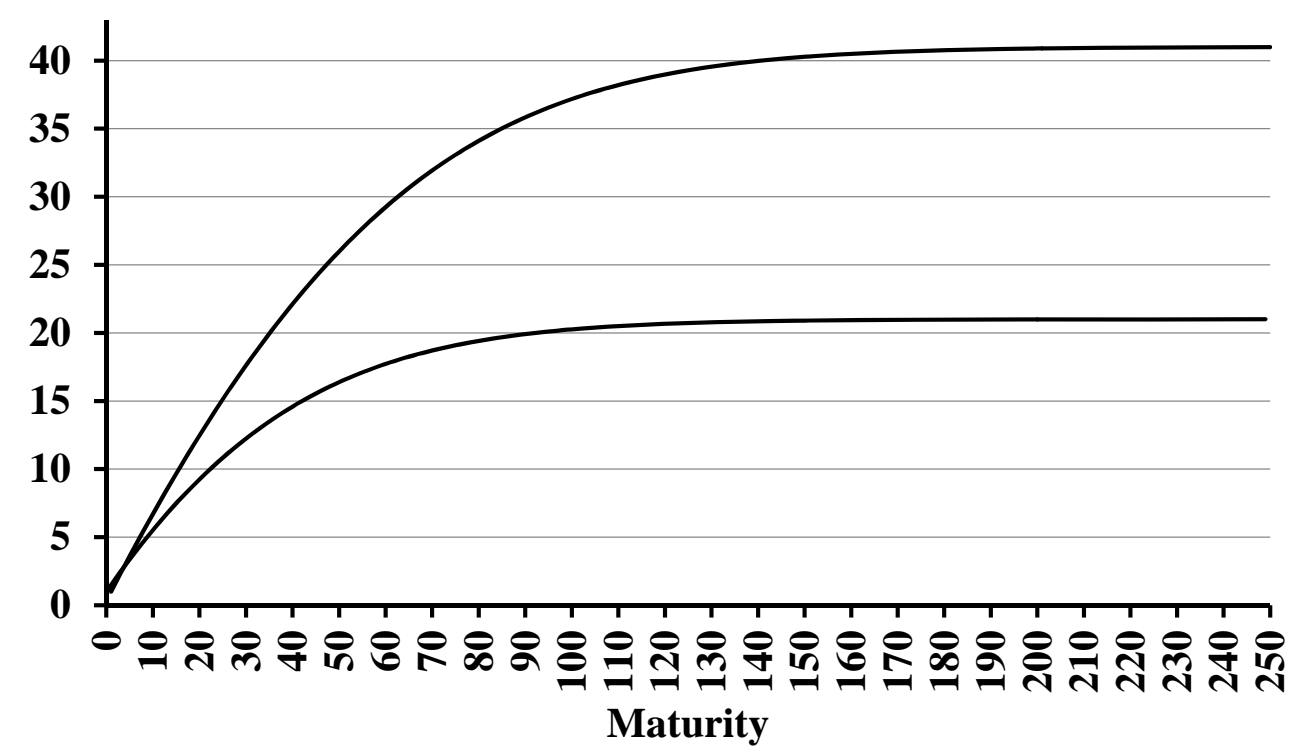

Figure 5: Duration measure as a function of time to maturity for a cash flow with constant absolute growth (upper graph) and an annuity (lower graph). For both graphs, $r=5 \%$.

Duration measures for higher order polynomial payment streams can be obtained by using this recursive procedure. The closed form expressions for finite maturities inevitably become longer and more complicated as the order of the polynomial increases.

\subsection{Duration measures for cyclically varying payment streams}

Duration measures for cyclically varying payment streams can be found using the first periodic differences, as was used for finding present values in Theorem 2. The resulting expressions, for $T$ representing a number of full cycles, are listed here; their derivations are found in A.4.

$$
\begin{aligned}
\frac{V(\underline{C} t ; r, T)}{V(\underline{C} ; r, T)}= & 2 \frac{(1+r)^{2}-(1+r) \cos (\omega)}{1+(1+r)^{2}-2(1+r) \cos (\omega)}-\frac{(1+r) \sin (\omega+\phi)}{(1+r) \sin (\omega+\phi)-\sin (\phi)}- \\
& \frac{T}{(1+r)^{T}-1} \\
\frac{V(\underline{C} t ; r, \infty)}{V(\underline{C} ; r, \infty)}= & 2 \frac{(1+r)^{2}-(1+r) \cos (\omega)}{1+(1+r)^{2}-2(1+r) \cos (\omega)}-\frac{(1+r) \sin (\omega+\phi)}{(1+r) \sin (\omega+\phi)-\sin (\phi)} .
\end{aligned}
$$


Example 5 (cont.): Alternating signs

For this case, $\omega=\pi$ and $\phi= \pm \frac{\pi}{2}$, depending upon whether the first element is positive or negative. However, the result for a full cycle is independent of the sign of the first element. By directly inserting these values into Equations (86) and (87) we have the following expression for the duration for an even value of $T$ :

$$
D_{(\omega, \phi)=\left(\pi, \pm \frac{\pi}{2}\right)}=\frac{1+r}{2+r}-T \frac{1}{(1+r)^{T}-1},
$$

with limiting value $(1+r) /(2+r)$ as $T \rightarrow \infty$. For smaller values of $T$, the duration is significantly negative, due to the denominator in the last term being small.

The duration measure for all values of $T$ is easily obtained in this simple case by differentiation of the price formula as given in Equation (61):

$$
D_{(\omega, \phi)=\left(\pi, \pm \frac{\pi}{2}\right)}=\frac{1+r}{2+r}+(-1)^{T-1} T \frac{1}{(1+r)^{T}-1},
$$

This case highlights the extreme nature of duration when cash flows are cyclical. The sign of the duration changes with the bond's position in the cycle, and duration can be quite large in absolute value even when the maturity is short. For example, for $r=5 \%$ and $T=2$ the duration is -19 , or approximately ten times the magnitude of the longest duration of a bullet bond with the same maturity and yield. Yet, for the same interest rate and $T=3$ the duration is 20.02, only slightly larger in absolute value (while also changing sign).

The absolute value of the duration for longer term maturities slowly decreases and tends to 0.5 , indicating a very modest exposure to interest rate risk. More elaborated examples of this is shown in the following, cf. the results in Table 1. 
Table 1: Combinations of value and duration for a quarterly varying cyclic payment stream around zero.

\begin{tabular}{|c|r|r|r|r|r|r|r|r|}
\hline & \multicolumn{5}{|c|}{$\omega=\frac{\pi}{2}, \phi=-\frac{\pi}{4}$} & \multicolumn{3}{c|}{$\omega=\frac{\pi}{2}, \phi=\frac{\pi}{4}$} \\
$T$ & \multicolumn{2}{|c|}{ Value } & \multicolumn{2}{c|}{ Duration } & \multicolumn{2}{c|}{ Value } & \multicolumn{2}{c|}{ Duration } \\
\hline & $r=1 \%$ & $r=5 \%$ & $r=1 \%$ & $r=5 \%$ & $r=1 \%$ & $r=5 \%$ & $r=1 \%$ & $r=5 \%$ \\
\hline \multirow{2}{*}{4} & 0.0275 & 0.1222 & -98.0050 & -18.0244 & 0.0001 & 0.0030 & -198.5025 & -38.5122 \\
20 & 0.1270 & 0.4296 & -90.3232 & -11.5605 & 0.0006 & 0.0105 & -190.8207 & -32.0483 \\
80 & 0.3862 & 0.6755 & -65.2433 & -1.1108 & 0.0019 & 0.0165 & -165.7409 & -21.5986 \\
$\infty$ & 0.7036 & 0.6895 & 0.5075 & 0.5366 & 0.0035 & 0.0168 & -99.9901 & -19.9512 \\
\hline
\end{tabular}

Payment streams that cycle around 0 may, as one example, be thought of as realizations of a multiple delivery forward contract ${ }^{18}$ where the underlying spot price varies cyclically. Such payment streams can have both high levels of duration and highly volatile duration, when looked upon in isolation; consequently, they can be very sensitive to interest rate changes as already demonstrated in Example 5 .

Table 1 shows combinations of value and duration for the case shown in the left ypanel of Figure 2 with quarterly frequency. The combinations span one, five and twenty cycles together with the asymptotic limits of these measures for interest rates of $1 \%$ and $5 \%$, respectively. We also show the numbers for a phase shift of one quarter, i.e. when $\phi=\pi / 4$ instead of $\phi=-\pi / 4$.

Table 1 reveals that the durations of cyclical cash flows are very large compared to what is conventionally found in bond markets and for most fixed income derivatives, at the time a new cycle is initiated. It also shows that the duration is negative at these times and that the convergence towards the asymptotic positive limit is quite slow. A closer examination of the duration values produced for other values of $T$ shows that they vary between these large negative values and relatively large positive values at even times that are not multiples of four. For odd time indices the numerical values of the duration are relatively small. This indicates that interest rate risk management is a delicate issue if one faces cyclically varying payment streams.

However, when cyclical cash flows are deviations from a more regular payment stream, e.g.,

\footnotetext{
${ }^{18}$ Such contracts are also called flow forward contracts and are similar to financial swap contracts. Fixed price contracts for delivery of electricity or natural gas are examples of this.
} 
an annuity at a certain level, this erratic behavior is soon dominated by the value and the duration of the annuity. The equivalent numbers for the following payment stream:

$$
C_{t}=1+\sin (\omega t+\phi)
$$

which still exhibits a large amplitude compared to the level of payments, are shown in Table 2 .

Table 2: Combinations of value and duration for a quarterly varying cyclic payment stream around one.

\begin{tabular}{|c|c|c|c|c|c|c|c|c|c|}
\hline \multirow[b]{2}{*}{$h$} & \multirow{3}{*}{$T$} & \multicolumn{4}{|c|}{$\omega=\frac{\pi}{2}, \phi=-\frac{\pi}{4}$} & \multicolumn{4}{|c|}{$\omega=\frac{\pi}{2}, \phi=\frac{\pi}{4}$} \\
\hline & & \multicolumn{2}{|c|}{ Value } & \multicolumn{2}{|c|}{ Duration } & \multicolumn{2}{|c|}{ Value } & \multicolumn{2}{|c|}{ Duration } \\
\hline & & $r=1 \%$ & $r=5 \%$ & $r=1 \%$ & $r=5 \%$ & $r=1 \%$ & $r=5 \%$ & $r=1 \%$ & $r=5 \%$ \\
\hline 1 & 4 & 3.9294 & 3.6682 & 1.7855 & 1.7571 & 3.9021 & 3.5489 & 2.4805 & 2.4047 \\
\hline 5 & 20 & 18.1725 & 12.8918 & 9.4673 & 8.2210 & 18.0462 & 12.4727 & 10.1623 & 8.8686 \\
\hline 20 & 80 & 55.2744 & 20.2720 & 34.5471 & 18.6709 & 54.8901 & 19.6129 & 35.2422 & 19.3182 \\
\hline$\infty$ & $\infty$ & 100.7036 & 20.6895 & 100.2969 & 20.3181 & 100.0035 & 20.0168 & 100.9928 & 20.9656 \\
\hline
\end{tabular}

\section{Convexity}

Analogous to the duration as a linear approximation to the present value function, the convexity $C$ is a quadratic approximation that captures the curvature of the present value function:19

$$
\begin{gathered}
C=\frac{\partial^{2} V(\underline{C} ; r)}{\partial r^{2}} \frac{(1+r)^{2}}{V(\underline{C} ; r)}=\frac{\sum_{t=1}^{T} C_{t}(t+1) t(1+r)^{-t}}{\sum_{t=1}^{T} C_{t}(1+r)^{-t}}=\sum_{t=1}^{T} \omega_{t}(t+1) t, \\
V(\underline{C} ; r+\Delta r, T) \simeq V(\underline{C} ; r, T)\left[1-D \frac{\Delta r}{1+r}+\frac{1}{2} C\left(\frac{\Delta r}{1+r}\right)^{2}\right] .
\end{gathered}
$$

\footnotetext{
${ }^{19}$ Some authors define the convexity as $\sum_{t=1}^{T} C_{t} t^{2}(1+r)^{-t} / V(\underline{C} ; r, T)$ instead of the definition given in Equation (91). Doing so, the interpretation in terms of a Taylor series expansion no longer holds.
} 
with $\omega_{t}$ defined as in Equation $72^{20}$

We explore the convexity measure for our common examples by finding the value of both the numerator and the denominator in (91). The general expressions relating to the cyclically varying payment streams are left out due to their complexity.

For polynomial payment streams, the convexity is the weighted sum of the convexities for payment streams of the form $t^{p}$, where we know $V\left(t^{p} ; r, T\right)$ from Theorem 1 ;

$$
\begin{aligned}
\frac{\partial^{2} V\left(t^{p} ; r, t\right)}{\partial r^{2}} \frac{(1+r)^{2}}{V\left(t^{p} ; r, T\right)} & =\frac{V\left(t^{p+1}(1+t) ; r, T\right)}{V\left(t^{p} ; r, T\right)} \\
\frac{V\left(\sum_{p=0}^{n} a_{p} t^{p+1}(t+1) ; r, T\right)}{V\left(\sum_{p=0}^{n} a_{p} t^{p} ; r, T\right)} & =\sum_{p=0}^{n} W_{p}\left[\frac{V\left(t^{p+2} ; r, T\right)+V\left(t^{p+1} ; r, T\right)}{V\left(t^{p} ; r, T\right)}\right] .
\end{aligned}
$$

Example 11(cont.): Convexity of the annuity

The convexity of an annuity, $C_{a n n}$. is obtained by inserting the by now known results in Equation (93) with $p=0$. Using the result found in Equation (38) and after some straightforward manipulations of terms we arrive at:

$$
\begin{aligned}
C_{a n n .} & =\frac{V\left(t^{2} ; r, T\right)+V(t ; r, T)}{V(1 ; r, T)} \\
& =\frac{\frac{1}{r^{2}}\left[(1+r)(2+r) V(1 ; r, T)-(1+r)^{-T} T(T+2(1+r))\right]+V(t ; r, T)}{V(1 ; r, T)} \\
& =2 \frac{1+r}{r} D_{\text {ann. }}-\frac{T(T+1)}{(1+r)^{T}-1} .
\end{aligned}
$$

A closer examination of this expression reveals that it is both an increasing and concave function of $T$. In the case of a perpetuity, the last terms disappears. Hence, the convexity of the perpetuity is $2((1+r) / r)^{2}$.

Example 2 (cont.): Convexity for a payment stream with a constant growth factor

Consider next the sequence of payments $C_{t}=t^{2} G_{t}$. Here the first differences are:

$$
\Delta_{t}=(t+1)^{2} G_{t+1}-t^{2} G_{t}= \begin{cases}G_{t}\left(g t^{2}+(2 t+1)(1+g)\right) & (\text { for } t \leq T-1) \\ -T^{2} G_{T} & (\text { for } t=T)\end{cases}
$$

\footnotetext{
${ }^{20}$ Analogous to the duration, the term $(1+r)^{2}$ is necessary in order to obtain the interpretation as a weighted sum. It disappears if continuous compounding is used.
} 
Using the same methodology as for $t G_{t}$, the result of Proposition 1 can be written as:

$$
\begin{aligned}
r V\left(t^{2} G_{t} ; r, T\right)= & 1+g V\left(t^{2} G_{t} ; r, T\right)-g T^{2} G_{T}(1+r)^{-T}+ \\
& 2(1+g) V\left(t G_{t} ; r, T\right)-2 T G_{T+1}(1+r)^{-T}+ \\
& (1+g) V\left(G_{t} ; r, T\right)-G_{T+1}(1+r)^{-T}-T^{2} G_{T}(1+r)^{-T} .
\end{aligned}
$$

By collecting terms to prepare for the calculation of the convexity we have:

$$
\begin{aligned}
& (r-g)\left[V\left(t^{2} G_{t} ; r, T\right)+V\left(t G_{t} ; r, T\right)\right]=1+(2+g+r) V\left(t G_{t} ; r, T\right)+ \\
& (1+g) V\left(G_{t} ; r, T\right)-G_{T+1}(1+r)^{-T}-\left(T^{2}+2 T\right) G_{T+1}(1+r)^{-T} .
\end{aligned}
$$

After dividing through with $(r-g) V\left(G_{t} ; r, T\right)=1-G_{T+1}(1+r)^{-T}$ and reorganizing terms we arrive at:

$$
\begin{aligned}
C_{\text {growth }} & =\frac{V\left(t^{2} G_{t} ; r, T\right)+V\left(t G_{t} ; r, T\right)}{V\left(G_{t} ; r, T\right)} \\
& =\frac{2+g+r}{r-g} D_{\text {growth }}+\frac{(1+r)}{r-g}-\left(T^{2}+2 T\right) \frac{G_{T+1}(1+r)^{-T}}{1-G_{T+1}(1+r)^{-T}} \\
& =2 \frac{1+r}{r-g} D_{\text {growth }}-\frac{T^{2}+T}{\left(\frac{1+r}{1+g}\right)^{T}-1} .
\end{aligned}
$$

The asymptotic limit is $2((1+r) /(r-g))^{2}$.

Example 3 (cont.): Convexity of the bullet bond

Analogous to the derivation of the duration, the convexity of a bullet bond is a weighted average of the convexities of a zero-coupon bond and an annuity with weights corresponding to their share of the total value ${ }^{21}$ For the zero-coupon bond, the convexity is straightforward to find from the definition, cf. (91), as $T(T+1)$. Hence, the convexity for a bullet bond with coupon rate $C, C_{\text {bullet }}$, is:

$$
C_{\text {bullet }}=\frac{C a_{\bar{T} \mid r}}{C a_{\bar{T} \mid r}+(1+r)^{-T}} C_{a n n .}+\frac{(1+r)^{-T}}{C a_{\bar{T} \mid r}+(1+r)^{-T}} T(T+1),
$$

\footnotetext{
${ }^{21}$ Closed form expressions for convexity in the sense given in footnote 19 are provided in Nawalkha and Lacey (1988) for the bullet bond. They also derived higher order measures, $\sum_{t=1}^{T} C_{t} t^{m}(1+r)^{-t} / V(\underline{C} ; r, T)$, for $m>2$ through a recursive relationship. In Nawalkha and Lacey $(1990,1991)$ closed form expressions for the annuity bond as well as an analysis of the socalled $M^{2}$ measure can be found.
} 


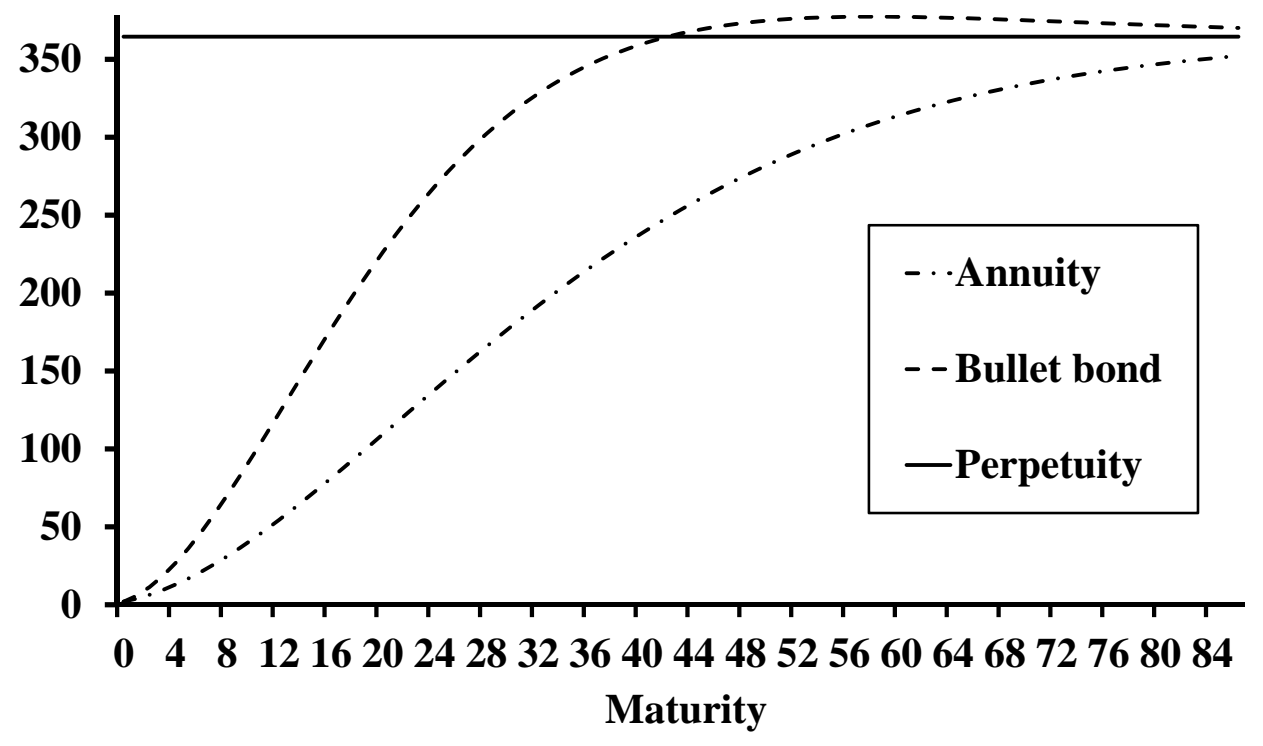

Figure 6: Convexity measure as a function of time to maturity for the annuity and the bullet bond with $c=4 \%$ and $r=8 \%$.

which after substitution and rewriting becomes:

$$
\begin{aligned}
C_{\text {bullet }}= & \frac{C a_{\bar{T} r}}{C a_{\bar{T} r}+(1+r)^{-T}} 2\left[\left(\frac{1+r}{r}\right)^{2}-\frac{1+r}{r} \frac{T(1+r)^{-T}}{1-(1+r)^{-T}}\right]+ \\
& \frac{(1+r)^{-T}}{C a_{\bar{T} \mid r}+(1+r)^{-T}} T(T+1)\left[1-\frac{C}{r}\right] \\
= & \frac{C a_{\bar{T} r}}{C a_{\bar{T} r}+(1+r)^{-T}} 2\left(\frac{1+r}{r}\right) D_{a n n .}+\frac{(1+r)^{-T}}{C a_{\bar{T} \mid r}+(1+r)^{-T}} T(T+1)\left[1-\frac{C}{r}\right] .
\end{aligned}
$$

Again, a simple manipulation of this formula shows that the convexity of a bullet bond is not a monotonic function of time to maturity $T$, when sold below par (i.e. when $r>c$ ). It will overshoot its asymptotic limit, which is equal to the convexity of the perpetual and found as the limiting value of the first term in 104.

Figure 6 shows - analogous to Figure 4 - the convexity measures for the annuity and the bullet bond. 
Example 4: Convexity for a payment stream with constant absolute growth

We state the result for the linear case with $a_{0}=0$. Then the convexity $C_{l i n}$. is

$$
\begin{aligned}
C_{\text {lin. }} & =\frac{V\left(t^{3}+t^{2} ; r, T\right)}{V(t ; r, T)} \\
& =\frac{\frac{(1+r)\left(r^{2}+6(1+r)\right)}{r^{3}} a_{T r r}-(1+r)^{-T}\left[\frac{r^{2} T^{3}+3 T^{2} r(1+r)+3 T(1+r)(2+r)}{r^{3}}\right]}{\frac{1+r}{r} a_{\overline{T r} r}-\frac{T}{r}(1+r)^{-T}}+D_{l i n .} .
\end{aligned}
$$

A reduction of this expression requires a few tedious, but straightforward arithmetic operations, with the final result:

$$
C_{\text {lin. }}=\frac{1}{r^{2}} \frac{2(1+r)^{2}(3+r) a_{\overline{T r} r}-(1+r)^{-T} T\left[r^{2} T(T+1)+r(1+r)(3 T+5)+6(1+r)\right]}{(1+r) a_{\overline{T r} r}-T(1+r)^{-T}} .
$$

The limiting value for $T \rightarrow \infty$ is:

$$
\frac{2(1+r)(3+r)}{r^{2}}
$$

Analytic expressions for higher order polynomials are increasingly complicated for finite maturities, whereas the expressions for infinite maturity remain analytically tractable.

\section{$7 \quad$ Summary of Major Findings}

This paper identifies new properties of the present value operator, which we refer to as the first difference property (FDP) and the second difference property (SDP), respectively. We show how they can be used to identify a large and potentially unlimited number of analytic expressions for present value and related measures such as duration and convexity.

While we are aware of earlier efforts to identify present value rules for a broad class of payment streams that are continuous in time over an infinite time horizon, we are not aware of similar efforts to identify analytic expressions for present value, duration and convexity for nontraditional cash flows that provide discrete time payments. Most practical applications are discrete in nature and also most often over a finite time horizon. This paper shows how the difference properties can be used to fill this gap.

For example, the present value rule is well known for a perpetual cash flow that grows at a uniform geometric rate, often referred to as Gordon's growth formula. However, we are 
not aware of prior efforts to identify corresponding rules for the duration and convexity of such a payment stream. By applying the difference operations we derive analytic expressions for present value, duration and convexity, including the finite time horizon case. We do the same for the case of a cash flow that grows each period by a constant dollar amount.

New analytic expressions are also identified for cash flows that exhibit cyclical variation over time. Such patterns are common for investments in agricultural products, for contracting in the energy sector and for the earnings of firms that rely heavily on seasonal activities and sales. Nevertheless, we are not aware of prior efforts to identify present value rules for these seemingly important cash flow patterns. We address this gap and derive analytic expressions for present value and duration for such payment streams. In the course of doing so we also demonstrate that such payment streams can be highly sensitive to interest rate risk as measured by their duration, which takes on numerically large values that also varies in sign over the cycle.

We also demonstrate how the deferral of capital gains taxation due to the realization principle affects the measured yield after tax. In this vein we also note that duration measures after tax have received very little attention in the literature; derivations of such measures is devoted to future research.

In addition to providing greater clarity with respect to the foundations for these and other present value rules, we hope the identification of new analytic expressions will help bridge the current gap between mechanical calculations of present value and the derived risk measures and the driving forces behind those calculations.

\section{References}

Abramowitz, M., and I. A. Stegun (eds.), 1984, Pocketbook of Mathematical Functions, Verlag Harri Deutsch, Thun, Frankfurt/Main.

Babcock, G. C., 1985, "Duration as a weighted average of two factors," Financial Analysts Journal, 41(2), March-April, 75-76.

Buser, S. A., and B. A. Jensen, 2017, "The First Difference Property of the Present Value Operator," The Quarterly Journal of Finance, 7(4).

Chua, J. H., 1984, "A Closed-Form Formula for Calculating Bond Duration," Financial Analysts Journal, 40(3), May-June, 76-78. 
Chua, J. H., 1985, "Calculating Bond Duration: Further Simplification," Financial Analysts Journal, 41(3), November-December, 76.

Chua, J. H., 1988, "A Generalized Formula for Calculating Bond Duration," Financial Analysts Journal, 44(3), May-June, 65-66.

Deacon, M., and A. Derry, 2004, Inflation-Indexed Securities, Wiley Finance, Hempstead, Great Britain.

Geman, H., and V.-N. Nguyen, 2005, "Soybean Inventory and Forward Curve Dynamics," Management Science, 51(7), 1076-1091.

Gordon, M. J., 1959, "Dividend Earnings and Stock Prices," Review of Economics and Statistics, 41(2), 99-105.

Gordon, M. J., and E. Shapiro, 1956, "Capital Equipment Analysis: The Required Rate of Profit," Management Science, 3(1), 102-110.

Hossack, I., and G. Taylor, 1975, "A generalization of Makeham's formula for valuation of securities," Journal of the Institute of Actuaries, 101, 89-95.

Jensen, B. A., 2013, "Makeham's formula: Some Applications in Fixed Income Analysis," working paper, Copenhagen Business School.

Macaulay, F., 1938, Some theoretical problems suggestes by the movements of interest rates, bond yields, and stock prices in the U.S. since 1856, NBER, New York, USA.

Magni, C. A., 2014, "Generalized Makeham's Formula and Economic Profitability," Insurance: Mathematics and Economics, 53(3), 747-756.

Makeham, W., 1875, "On the solution of problems connected with loans repayable by instalments," Journal of the Institute of Actuaries, 18, 132-143.

Nawalkha, S. K., and N. J. Lacey, 1988, "Closed-Form Solutions of Higher-Order Duration Measures," Financial Analysts Journal, 44(6), Nov-Dec, 82-84.

Nawalkha, S. K., and N. J. Lacey, 1990, "Closed-Form Solutions of Convexity and M-Square," Financial Analysts Journal, 46(1), Jan-Feb, 75-77.

Nawalkha, S. K., and N. J. Lacey, 1991, "Convexity for Bonds with Special Cash Flow Streams," Financial Analysts Journal, 47(1), Jan-Feb, 80-82. 
Redington, F., 1952, "Review of the principles of life-office valuations," Journal of the Institute of Actuaries, 78(3), 286-340.

Rodriguez, R. j., 1988, "Investment Horizon, Taxes and Maturity Choice for Discount Coupon Bonds," Financial Analysts Journal, 44, September-October, 67-69.

Roll, R., 1984, "After-tax investment results from long-term vs. short-term discount coupon bonds," Financial Analysts Journal, 39, January-February, 43-54.

Samuelson, P. A., 1964, "Tax Deductibility of Economic Depreciation to Insure Invariant Valuations," Journal of Political Economy, 72(6), 604-606.

Sørensen, C., 2002, "Modeling seasonality in agricultural commodity futures," The Journal of Futures Markets, 22(5), 393-426.

Williams, J. B., 1938, The Theory of Investment Value, North Holland Publishing Company, Amsterdam.

\section{A Proofs}

\section{A.1 Proof of Proposition 1}

The general expression for the present value of a cash flow in Equation (2) is defined as of time 0 . By multiplying this equation by $(1+r)$ we effectively shorten the term to maturity for each component of the cash flow by one period. As a result, Equation (108) identifies the present value of the cash flow as of time 1 :

$$
\begin{aligned}
(1+r) V(\underline{C} ; r, T) & =\sum_{t=1}^{T} C_{t}(1+r)^{-(t-1)}=C_{1}+\sum_{t=2}^{T} C_{t}(1+r)^{-(t-1)} \\
& =C_{1}+\sum_{t=1}^{T} C_{t+1}(1+r)^{-t}
\end{aligned}
$$

In the last sum we have made a parallel shift of the summation index $t$ and simultaneously made use of the convention that $C_{T+1}=0$ for a payment stream with finite time horizon $T$. In this way we can change the upper limit in this summation to $T$ instead of $T-1$ without changing the sum - it just means adding a zero. 
By subtracting Equation (2) from (108) we establish that the change in present value from time 0 to time 1 is equal to the cash flow at time 1 plus the present value of the period by period change in future cash flows:

$$
r V(\underline{C} ; r, T)=C_{1}+\sum_{t=1}^{T}\left(C_{t+1}-C_{t}\right)(1+r)^{-t}=C_{1}+\sum_{t=1}^{T} \Delta_{t}(1+r)^{-t} .
$$

The proof is the same when $T=\infty$; here both the values $T$ and $T-1$ in the upper summation limit must be read as $\infty$.

\section{A.2 Proof of Theorem 1}

For an infinite horizon polynomial payment stream $(T=\infty)$ we have $\forall t$ that:

$$
\Delta_{t}=(t+1)^{n}-t^{n}=\sum_{p=0}^{n-1}\left(\begin{array}{l}
n \\
p
\end{array}\right) t^{p}
$$

Hence,

$$
\sum_{t=1}^{\infty} \Delta_{t}(1+r)^{-t}=\sum_{t=1}^{\infty} \sum_{p=0}^{n-1}\left(\begin{array}{l}
n \\
p
\end{array}\right) t^{p}(1+r)^{-t}, \quad t=0,1,2, \ldots, T-1 .
$$

Changing the order of summation results in (35).

For the finite horizon case, we have that:

$$
\begin{aligned}
& \Delta_{t}=(t+1)^{n}-t^{n}=\sum_{p=0}^{n-1}\left(\begin{array}{l}
n \\
p
\end{array}\right) t^{p}, t=1,2, \ldots, T-1 \\
& \Delta_{T}=-T^{n} .
\end{aligned}
$$

Inserting these into Equation (4) we have:

$$
\begin{aligned}
V\left(t^{n} ; r, T\right) & =\frac{1}{r}\left[1+\sum_{t=1}^{T} \Delta_{t}(1+r)^{-t}\right] \\
& =\frac{1}{r}\left[1+\sum_{t=1}^{T-1} \sum_{p=0}^{n-1}\left(\begin{array}{l}
n \\
p
\end{array}\right) t^{p}(1+r)^{-t}-T^{n}(1+r)^{-n}\right] \\
& =\frac{1}{r}\left[1+\sum_{p=0}^{n-1} \sum_{t=1}^{T-1}\left(\begin{array}{l}
n \\
p
\end{array}\right) t^{p}(1+r)^{-t}-T^{n}(1+r)^{-n}\right],
\end{aligned}
$$


which proves Equation (36).

\section{A.3 Proof of Theorem 2}

In the setting with cyclical variations, the first differences $C_{t+1}-C_{t}$, as otherwise used in this paper, are not generally useful. In order to arrive at a general result for such payment streams, the first difference property must be generalized to a situation where the differences are taken over full cycles as $C_{t+h}-C_{t}$ instead of just neighboring payments. These first periodic differences vanish by construction, except for the terminal terms in the finite horizon case. The first term, $C_{1}$, which was a key ingredient in the present value formula in Proposition 1, must be replaced with the future value of the first cycle.

The proof makes use of the following three ingredients:

- the discrete compounding interest rate $r$ is converted into its exponentially compounding counterpart $r_{c}$ through the relationship $(1+r)^{-t}=e^{r_{c} t}$

- the Euler representation of the sine function in terms of complex exponentials

- the standard rule for a sum of geometrically growing terms

We apply these ingredients through the following steps:

$$
\begin{aligned}
& \sum_{t=1}^{T} \sin (\omega t+\phi)(1+r)^{-t}=\sum_{t=1}^{T} \frac{e^{i(\omega t+\phi)}-e^{-i(\omega t+\phi)}}{2 i} e^{-r_{c} t}= \\
& \sum_{t=1}^{T} \frac{e^{\left(i \omega-r_{c}\right) t+i \phi}-e^{-\left(i \omega+r_{c}\right) t-i \phi}}{2 i}=\frac{1}{2 i}\left[e^{i \phi} \frac{1-e^{\left(i \omega-r_{c}\right) T}}{e^{-\left(i \omega-r_{c}\right)}-1}-e^{-i \phi} \frac{1-e^{-\left(i \omega+r_{c}\right) T}}{e^{\left(i \omega+r_{c}\right)}-1}\right]= \\
& \frac{1}{2 i}\left[\frac{e^{i \phi}\left(1-e^{\left(i \omega-r_{c}\right) T}\right)\left(e^{\left(i \omega+r_{c}\right)}-1\right)-e^{-i \phi}\left(1-e^{-\left(i \omega+r_{c}\right) T}\right)\left(e^{-\left(i \omega-r_{c}\right)}-1\right)}{\left(e^{-\left(i \omega-r_{c}\right)}-1\right)\left(e^{\left(i \omega+r_{c}\right)}-1\right)}\right]= \\
& \frac{1}{2 i}\left[\frac{-\left(e^{i \phi}-e^{-i \phi}\right)+\left(e^{i(\phi+\omega)+r_{c}}-e^{-i(\phi+\omega)+r_{c}}\right)}{1+e^{2 r_{c}}-2 e^{r_{c}}\left(\frac{e^{i \omega}-e^{-i \omega}}{2}\right)}\right]+ \\
& \frac{1}{2 i}\left[\frac{e^{-r_{c} T}\left(e^{i(\omega T+\phi)}-e^{-i(\omega T+\phi)}\right)-e^{-r_{c}(T-1)}\left(e^{i(\omega(T+1)+\phi)}-e^{-i(\omega(T+1)+\phi)}\right)}{1+e^{2 r_{c}}-2 e^{r_{c}}\left(\frac{e^{i \omega}-e^{-i \omega}}{2}\right)}\right] .
\end{aligned}
$$

Reverting back to the sine and cosine functions and the discrete compounding gives Equation (66). 


\section{A.4 Duration measures in subsection 5.2}

In order to derive the duration measures in Equations 86 and 87, we use the following modified the FDP approach, where differences are taken over the number of time steps $h$ required to complete the first full cycle instead of over one time step:

$$
\begin{aligned}
& V(\underline{C} t ; r, T)=\sum_{t=1}^{T} C_{t} t(1+r)^{-t} \\
& (1+r)^{h} V(\underline{C} t ; r, T)=\sum_{t=1}^{h} C_{t} t(1+r)^{-(t-h)}+\sum_{t=h+1}^{T} C_{t} t(1+r)^{-(t-h)} \\
& =\sum_{t=1}^{h} C_{t} t(1+r)^{-(t-h)}+\sum_{t=1}^{T-h} C_{t+h}(t+h)(1+r)^{-t} \\
& {\left[(1+r)^{h}-1\right] V(\underline{C} t ; r, T)=\sum_{t=1}^{h} C_{t} t(1+r)^{-(t-h)}+\sum_{t=1}^{T}\left[\left(C_{t+h}-C_{t}\right)(t+h)+C_{t} h\right](1+r)^{-t}} \\
& =\sum_{t=1}^{h} C_{t} t(1+r)^{-(t-h)}-\sum_{t=T+1-h}^{T} C_{t}(t+h)(1+r)^{-t}+ \\
& h \sum_{t=1}^{T} C_{t}(1+r)^{-t} \\
& r a_{\bar{h} \mid r} \frac{V(\underline{C} t ; r, T)}{V(\underline{C} ; r, T)}=\frac{\sum_{t=1}^{h} C_{t} t(1+r)^{-t}}{V(\underline{C} ; r, T)}+h(1+r)^{-h}- \\
& (1+r)^{-T} \frac{\sum_{j=1}^{h} C_{t}(T+j)(1+r)^{-j}}{V(\underline{C} ; r, T)} \\
& r a_{\bar{h} r} \frac{V(\underline{C} t ; r, T)}{V(\underline{C} ; r, T)}=r a_{\bar{T} \mid r} \frac{\sum_{t=1}^{h} C_{t} t(1+r)^{-t}}{V(\underline{C} ; r, T)}+h(1+r)^{-h}- \\
& (1+r)^{-T} T \frac{\sum_{j=1}^{h} C_{t}(1+r)^{-j}}{V(\underline{C} ; r, T)} \text {. }
\end{aligned}
$$

To complete this part of the calculation we observe the following relationship, known as the equivalent annual annuity (EAA) or the equivalent annual cost (EAC):

$$
V(\underline{C} ; r, T)=V(\underline{C} ; r, h) a_{\bar{h} \mid r}^{-1} a_{\bar{T} \mid r} .
$$


After having inserted and collected terms we arrive at:

$$
\frac{V(\underline{C} t ; r, T)}{V(\underline{C} ; r, T)}=\frac{\sum_{t=1}^{h} C_{t} t(1+r)^{-t}}{V(\underline{C} ; r, h)}+h \frac{(1+r)^{-h}}{1-(1+r)^{-h}}-T \frac{(1+r)^{-T}}{1-(1+r)^{-T}} .
$$

In order to find the duration of the first cycle, we resort to the following mathematical fact, applied to Equation (68). Setting

$$
A=1-(1+r)^{-h}, B=(1+r) \sin (\omega+\phi)-\sin (\phi), C=1+(1+r)^{2}-2(1+r) \cos (\omega),
$$

we observe that the expression in Equation (68) is of the form $A B / C$. The duration of this, which is the first term in Equation $(122)$, is the logarithmic derivative ${ }^{22}$ scaled by $1+r$, cf. Equation (72), we can employ the following general rule:

$$
V=\frac{A B}{C} \Rightarrow V_{r}^{\prime}=\frac{A_{r}^{\prime} B+A B_{r}^{\prime}}{C}-\frac{A B}{C} \frac{C_{r}^{\prime}}{C} \Rightarrow \frac{V_{r}^{\prime}}{V}=\frac{A_{r}^{\prime}}{A}+\frac{B_{r}^{\prime}}{B}-\frac{C_{r}^{\prime}}{C} .
$$

Proceeding we obtain:

$$
\begin{gathered}
-(1+r) \frac{\frac{\partial}{\partial r}\left(1-(1+r)^{-h}\right)}{\left(1-(1+r)^{-h}\right)}=-\frac{h(1+r)^{-h}}{\left(1-(1+r)^{-h}\right)} \\
-(1+r) \frac{\frac{\partial}{\partial r}((1+r) \sin (\omega+\phi)-\sin (\phi))}{(1+r) \sin (\omega+\phi)-\sin (\phi)}=-\frac{(1+r) \sin (\omega+\phi)}{(1+r) \sin (\omega+\phi)-\sin (\phi)} \\
-(1+r) \frac{\frac{\partial}{\partial r}\left(1+(1+r)^{2}-2(1+r) \cos (\omega)\right)}{1+(1+r)^{2}-2(1+r) \cos (\omega)}=-2 \frac{(1+r)^{2}-(1+r) \cos (\omega)}{1+(1+r)^{2}-2(1+r) \cos (\omega)},
\end{gathered}
$$

which after substitution in Equation (122) proves the result in Equation (86). The result for the infinite horizon case is simply the limiting value as $T \rightarrow \infty$.

\footnotetext{
${ }^{22}$ In this particular setting we resort to differentiation, despite the intention to avoid such calculations as outlined in the introduction. However, the calculations involving differentiations are, indeed, quite straightforward.
} 